\title{
Using Population Genetics to Guide Malaria Vaccine Design
}

\author{
Alyssa E. Barry ${ }^{1,2}$, James Beeson'3 , John C. Reeder ${ }^{3,4}$, \\ Freya J.I. Fowkes ${ }^{3}$ and Alicia Arnott ${ }^{3}$ \\ ${ }^{1}$ Walter and Eliza Hall Institute for Medical Research, \\ 2Department of Medical Biology, University of Melbourne, \\ ${ }^{3}$ Burnet Institute, \\ ${ }^{4}$ Monash University, \\ Australia
}

\section{Introduction}

A broadly effective malaria vaccine is a missing component of the practical tools needed to control and eventually eliminate malaria. For more than 50 years malaria researchers have been searching for a strategy that provides long lasting protection against a broad range of parasite strains. Many different approaches have been tested including whole parasite and subunit vaccines composed of one or more parasite surface proteins that are naturally targeted by the host immune response.

Candidates in the malaria vaccine pipeline include surface exposed proteins from each of the morphologically distinct developmental stages of the parasite lifecycle within the human host. Many of these antigens have undergone rigorous developmental and preclinical testing as subunit vaccines [1] but only a few have reached advanced clinical trials: one reason being a lack of funding to carry all promising vaccine candidates to trial [2]. For candidates that have reached Phase II clinical trials in malaria endemic areas, there has been variable success. This is not surprising given that the malaria parasite is a complex and rapidly evolving organism that can quickly adapt to its ever-changing environment and effectively evade human immune responses. Limited understanding of the precise mechanisms and minimal requirements for antimalarial immunity has also hampered vaccine progress [3]. Moreover, the high degree of diversity of parasite surface antigens [4] and the allelic-specificity of the immune response [5,6] is likely to have contributed significantly to the variable success of malaria vaccines. However genetic diversity is often overlooked in vaccine design with most vaccines formulated on the basis of a single strain. It is now increasingly recognized that to be effective against the worldwide parasite population, a malaria vaccine may need to contain multiple variants of the target antigen [7].

Many studies have investigated the genetic diversity of vaccine antigens that are circulating in natural parasite populations but very little of this knowledge has been applied to malaria vaccine design. Typically, alleles from one or two reference strains (3D7 or FVO) are used in 
vaccine formulations, but this only partially represents the diversity of alleles circulating in the parasite population (Figure 1 [8]). Moreover, if vaccine alleles are found at low frequencies, the power to measure the success of vaccine trials is limited. This chapter reviews the current state of knowledge of the genetic diversity of leading malaria vaccine candidates, including those for both major human malaria parasites, Plasmodium falciparum and Plasmodium vivax. We examine the relevance of this diversity to malaria vaccines currently in development and how to use this information in determining which alleles should be used to provide broad coverage against the majority of parasite strains. Using leading candidate vaccine antigens as examples, we outline how population genetic tools can be used to characterise genetic diversity and the distribution of alleles and to define potentially immunologically relevant subgroups of alleles and polymorphisms. Finally, we make recommendations for the design of the next generation of malaria vaccines.

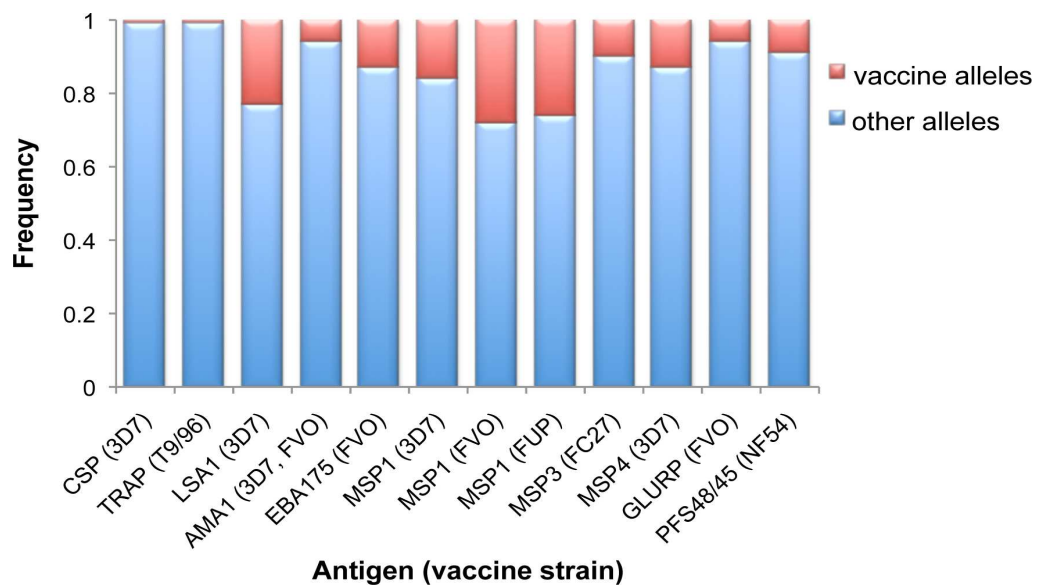

Fig. 1. Worldwide frequency of alleles represented in ten leading malaria vaccine candidates (adapted from [8]). $\mathrm{MSP}_{19} 3 \mathrm{D} 7$ and $\mathrm{FVO}$ are being developed as a combination vaccine as well as in isolation.

\section{Malaria vaccines: A brief history}

\subsection{Early studies}

The idea that a malaria vaccine might be possible was raised in the late 1800's with the observations of Robert Koch during his expedition to the Melanesian Island of Papua New Guinea (PNG). He found that while local children frequently displayed symptoms of malarial disease and harboured parasites in their blood, adults remained relatively disease and parasite-free [9]. He also noted that Malay and Chinese workers that had recently immigrated to PNG became ill with malaria more often than their counterparts who had been in residence for longer periods of time. These observations form the basis for our contemporary understanding of the epidemiology of malaria and the theory that immunity to malaria is eventually acquired in areas where people are constantly exposed to infection. In his conclusions, Dr. Koch stated that it would be possible to vaccinate against malaria toxins (and therefore the symptoms caused by infection). 
Some 60 years later, the feasibility of a human malaria vaccine received a boost when Sir Ian MacGregor demonstrated that immunity was conferred by transferring hyperimmune sera (IgG) from adults to at-risk African children [10]. Soon afterward, a team at New York University (NYU) including Ruth Nussenzweig and Jerome Vanderberg showed that mice could be vaccinated against malaria with irradiated Plasmodium bergei sporozoites [11]. In 1973, Clyde and colleagues at the University of Maryland in collaboration with the NYU team demonstrated that infections of humans via the bite of irradiated sporozoite-positive mosquitoes protected against subsequent homologous challenge, thus providing the first real evidence that man could be vaccinated against $P$. falciparum malaria [12] and subsequently, against $P$. vivax [13]. Later work showed that this approach was also effective in protecting against heterologous strains of P. falciparum [14]. These studies demonstrated that a malaria vaccine could elicit sterile protective immunity, and provided the reagents and tools to identify molecules that were important immune targets, such as the circumsporozoite surface protein (CSP) [15]. Unfortunately, at the time it was impractical to vaccinate large numbers of people with irradiated sporozoites isolated from infected mosquitoes and attempts to culture sporozoites showed only limited success [16].

\subsection{Subunit vaccines}

The increasing availability of molecular cloning and PCR techniques in the early 1980's saw a major shift in focus to the development of synthetic "subunit" vaccines based on individual parasite proteins and peptides and the abandonment of whole-parasite approaches. Several teams around the world had identified parasite surface proteins as targets such as the sporozoite antigens: circumsporozoite surface protein (CSP) [15] and thrombospondin related antigen (TRAP, $[17,18])$; Liver stage antigen 1 (LSA1,[19]); and the merozoite surface proteins, MSP1 [20], MSP2 [21], S-Antigen [22] and Apical Membrane Antigen 1 (AMA1, [23, 24]). Initial studies in animal models were promising but excitement began to wane as subunit vaccines showed only around half of the candidates had a protective effect in clinical trials. Of the eight malaria vaccine candidate antigens that have reached Phase II trials, only one, "RTS,S" which is based on CSP, has now been tested in Phase III clinical trials [25].

\subsection{Whole parasite vaccines}

The malaria parasite is a complex organism with 3000 or so known proteins [26, 27] including a few hundred that are immunogenic surface antigens [28]. This makes it a formidable target and therefore a vaccine as complex as the parasite itself may be required to immunise against malaria [29]. Evidence that sporozoite vaccinees were resistant to both homologous and heterologous challenge [14] makes whole-parasite vaccination approaches an appealing option so long as technological challenges can be overcome. New techniques for mass-producing sporozoites are reportedly in development [30] and both irradiation [31] and genetically attenuated $[32,33]$ parasite vaccines are being tested. Low doses of blood stage parasites have been shown to induce $\mathrm{T}$-cell mediated responses targeted at cytoplasmic proteins with high levels of sequence conservation [34]. In addition, chemicallyand genetically-attenuated blood-stage parasites are being developed (personal communication M. Good, Griffith University and K. Evans, Walter and Eliza Hall Institute). Consequently, whole parasite approaches have been experiencing a renaissance and have 
garnered much support from funding organisations [35]. As both subunit and whole parasite approaches are valid approaches $[35,36]$, they are being pursued in parallel. This review focuses primarily on the former approach.

\subsection{Malaria vaccine subclasses}

Malaria vaccines fall into three main classes based on the lifecycle stage that they target and their expected biological effects [37-39]. These include:

i. Pre-erythrocytic vaccines - based on antigens on the surface of the sporozoite or liver stage parasites or whole parasite (sporozoite approaches). These vaccines are designed to target the pre-erythrocytic stages of the parasite lifecycle and therefore inhibit infection.

ii. Blood stage vaccines - based on antigens on the surface of the blood stages such as the merozoite or intraerythrocytic stages or whole blood stage parasites. These diseaseblocking vaccines are designed to control parasitaemia by preventing invasion of uninfected erythrocytes (merozoite targets) or to control disease by preventing cytoadhesion (intraerythrocyte targets), which leads to pathogenesis.

iii. Transmission blocking vaccines - based on antigens on the surface of the sexual stages, expressed either within the human host or anopheline vector. Known as transmissionblocking vaccines because they are designed to elicit antibodies within the human host that will target gametocytes (transmission forms) or to be taken up in the mosquito blood meal to target parasite proteins within the mosquito midgut.

Vaccine approaches currently being tested include individual candidates from a single lifecycle stage as well as combination vaccines formulated with targets from multiple lifecycle stages.

\section{Subunit vaccine candidate antigens}

The advent of recombinant DNA technology has greatly facilitated the development of subunit malaria vaccines by providing the tools with which to synthesise large amounts of parasite protein, and to disrupt gene expression for detailed functional characterisation [4042]. Preclinical development for many candidate antigens has been successful and Phase II clinical trials have proceeded for at least eight candidate antigens, with evidence of antimalarial efficacy for MSP2 [6], CSP [43, 44], MSP3 [45] and AMA1 [46].

The release of the first full malaria genome sequence in 2002 allowed the systematic identification of novel malaria vaccine candidates amongst approximately 5300 genes. Genome wide screening for single nucleotide polymorphisms (SNPs) has been used to reveal loci under positive selection and therefore encoding proteins that may be targeted by the immune response [4, 47]. Proteomics approaches have characterised the "immunome" [48], and measured the abundance of parasite proteins on the parasite surface [49]. A pipeline of bioinformatic screens has also been used to identify surface proteins, gene knockouts and high-throughput immunological assays to identify novel surface antigens (e.g. [50]). In the last 10 years, the list of potential malaria vaccine candidates has rapidly expanded to encompass many antigens about which there is still much to be learnt. Targets that were discovered first are therefore further down the development pipeline rather than novel proteins that may ultimately be more successful. 
Below, we describe the most developed malaria vaccine candidates for the two major malaria parasites, $P$. falciparum and $P$. vivax as well as some novel vaccine candidates that have recently been identified for $P$. falciparum. This is not a comprehensive listing, a complete list of preclinical, clinical and inactive or discontinued malaria vaccine projects can be found in the World Health Organisation's Malaria Vaccine Rainbow Tables [51].

\subsection{Plasmodium falciparum}

P. falciparum is responsible for most of the mortality and morbidity associated with malaria, with up to 1 million deaths and around 225 million clinical cases caused by this species [52]. It is the only major human malaria parasite for which an in vitro culture system is available thus making it more tractable for investigations to characterise molecular structure and function and interactions with its human and anophelene hosts. As a result, malaria research has focused on developing ways to combat this particular species with less focus on other human infecting malaria parasites. The number of well-developed $P$. falciparum vaccine candidates in advanced stages of clinical development reflects this bias and there are strong candidates for every stage of the P. falciparum lifecycle within the human host. Some of these are described in detail below.

\subsubsection{Circumsporozoite Protein (CSP)}

CSP, which is found in the outer sporozoite membrane, was shown to be a target of antibodies that prevented hepatocyte infection, thereby interrupting an obligatory stage in the parasite's lifecycle [15]. Cloning of the CSP gene (amongst other antigens) opened the doors to mass production and it was hoped, a malaria vaccine [53]. CSP contains a central (NANP) repeat region containing B-cell epitopes flanked by non-repetitive sequences containing T-cell epitopes [53-55]. In 2004, a considerable protective effect was observed with a CSP-based vaccine known as RTS,S with $49 \%$ efficacy against severe malaria for a period of 18 months in children aged 1-4 years [44]. This vaccine is composed of the central repeat and the C-terminal regions of CSP isolated from the reference strain, 3D7, combined with a Hepatitis B surface antigen (which provides protection against Hepatitis B and therefore satisfies ethical requirements). Recently published data from a phase III trial including 6000 African children has shown a 50\% reduction in clinical episodes and 35\% reduction in severe disease in children aged 5-18 months [25]. Therefore, RTS,S is likely to be the first licensed malaria vaccine and should be available within the next few years [56]. Most would agree though that it is an important proof of principle rather than an end to the hunt for a vaccine.

\subsubsection{Thrombospondin Related Adhesion Protein (TRAP)}

TRAP also known as Sporozoite Surface Protein (SSP) [18, 57] is present within the micronemes of the sporozoite and is essential for gliding motility and host cell invasion in the mosquito and human pre-erythrocyte stages [58-60]. TRAP is also expressed in the blood stages though its function in this stage is not well understood [18, 57]. TRAP is a Type I transmembrane protein consisting of multiple adhesive domains and a central repeat region flanked by unique sequence. Anti-repeat monoclonal antibodies have been shown to at least partially inhibit sporozoite invasion in vitro [61] and potent T-cell responses have been identified in individuals vaccinated with irradiated sporozoites $[62,63]$ as well as people 
who are naturally exposed to malaria [64]. TRAP is highly polymorphic [65] and has also been shown to be under strong diversifying selection suggesting that it is a natural immune target [66].

Mapping of inhibitory epitopes has led to the development of the multiepitope (ME)-TRAP DNA vaccine, which contains 15 TRAP T-cell epitopes. The vaccine has been delivered using a prime-boost regime involving three doses of naked DNA or DNA fused to a viral vector, to produce potent T-cell responses in both animals and humans [67]. While safety and immunogenicity trials in the UK have shown promising results [68, 69], Phase II efficacy trials conducted in semi-immune adults in The Gambia and children in Kenya provided no evidence of protective efficacy [70-73].

\subsubsection{Apical Membrane Antigen 1 (AMA1)}

AMA1 is an $83 \mathrm{kDa}$ integral membrane protein expressed late in the asexual stages and found in the micronemes at the apical tip of merozoites [74]. Prior to schizont rupture the AMA1 prodomain is cleaved to reveal a $66 \mathrm{kDa}$ protein containing three subdomains (domains I, II and III) defined by their disulfide bonds [24]. AMA1 then relocates to the parasite surface [75] where further processing during invasion leads to shedding of two fragments of 44 and $48 \mathrm{kDa}$ whilst the $22 \mathrm{kDa}$ cytoplasmic tail remains in the membrane and is carried into the invaded erythrocyte [76, 77]. AMA1 is now known to function in formation of the tight junction through interactions with rhoptry neck proteins $[78,79]$. This molecule is also expressed in sporozoites where it is involved in hepatocyte invasion [80].

Antibodies against AMA1 are found in people living in malaria endemic areas [81-83], and these have been shown to block the invasion process [84-86]. The potential of AMA1 as a malaria vaccine candidate has been demonstrated in rodent models of malaria with both strain-specific [87-89] and cross-protective [90] protection observed. High levels of polymorphism in AMA1 [8, 91, 92] due to strong balancing selection [93] has resulted in hundreds of distinct AMA1 haplotypes; this might indicate that the development of a broadly effective AMA1-based malaria vaccine will be difficult. However, little is known about the antigenic diversity of AMA1 and recent studies suggest that immunization with a small number of different alleles might give broad reactivity [94, 95]. The availability of a 3D structural model for AMA1 has greatly advanced our understanding of antibody inhibition of AMA1 function by demonstrating that several polymorphisms are found on the edge of a hydrophobic pocket within which it is thought the receptor binds [96, 97]. A cluster of polymorphisms in this region, known as the "C1L cluster" contributes to immune escape [97], but the importance of residues outside this cluster remains unclear [92].

Vaccine candidates comprising full length AMA1 originating from the 3D7 strain alone (FMP2.1, [46] and in combination with FVO (AMA1-c1, [98]), have had variable success in Phase II efficacy trials. FMP2.1 demonstrated no efficacy using the primary endpoint of a clinical malaria episode, however when the secondary endpoint of clinical infections carrying the vaccine allele was defined, a substantial efficacy of $68 \%$ above that of the control was revealed [46]. The alternative vaccine candidate, AMA1-C1, showed no allelespecific efficacy however the prevalence of the vaccine allele amongst the samples was so low that sample numbers precluded a proper assessment of any effect [98]. Additionally, the adjuvant used was alum, which is less potent. 
Another approach for AMA1 that is being tested is a diversity-covering vaccine (PfAMA1 DiCo) whereby 355 published sequences were aligned and common polymorphisms (i.e. those found in $>16 \%$ of sequences) were used to design three synthetic constructs, which together encompass $97 \%$ of the sequence diversity [99]. So far there have not been any clinical trials however broader parasite growth inhibitory activity was observed in animals vaccinated with the DiCo mix than those vaccinated with native AMA1 from the FVO strain [99].

\subsubsection{Merozoite Surface Protein 1 (MSP1)}

MSP1 is the most abundant and best studied of the P. falciparum merozoite surface antigens. The protein is synthesised as a 190-kDa-precursor protein that is cleaved into four fragments of $83,30,38$, and $42 \mathrm{kDa}$ [100]. These exist as a non-covalently associated complex tethered by a glycosylphosphatidylinositol (GPI) anchor at the C-terminal [101]. During erythrocyte invasion, the N-terminal fragments are shed when $\mathrm{MSP}_{42}$ undergoes a secondary processing event generating a further $33 \mathrm{kDa}\left(\mathrm{MSP}_{33}\right)$ and $19 \mathrm{kDa}$ fragment $\left(\mathrm{MSP}_{19}\right)$ [102104] which facilitates parasite entry into the erythrocyte [105]. The $M S P 1_{19}$ fragment is retained on the surface of the invading merozoite [106].

The C-terminal end of MSP1 is a leading malaria vaccine candidate. The MSP1 $1_{33}$ sequence is dimorphic, with diverse alleles clustering within the two families known as K1 and Mad20 [107], however $\mathrm{MSP}_{19}$ is relatively conserved across the two allele families with six nonsynonymous SNPs that are commonly used to describe $\mathrm{MSP}_{19}$ haplotypes [108-113]. Within each of the allele-families, $\mathrm{MSP}_{33}$ also contains several single nucleotide polymorphisms (SNPs) and a 3 bp deletion $[108,111]$.

The FMP1/ASO2A vaccine, which is formulated with the $3 \mathrm{D} 7$ allele of $\mathrm{MSP}_{42}$, has been tested in Phase II trials. The vaccine initially showed promise in safety and immunogenicity trials in 40 Malian adults, with responses generated to parasite clones carrying diverse $\mathrm{MSP}_{42}$ alleles (FVO and Camp/FUP) [114]. Phase II efficacy trials including 400 children in Western Kenya did not protect against infection or lower parasite densities, nor did it reduce clinical episodes [115]. Though the authors stated that the vaccine was no longer a promising vaccine candidate, this was without having investigated how many vaccinees were infected with the vaccine strain. In addition, high throughput genotyping studies in Mali have demonstrated that the 3D7 allele had a prevalence of only 16\% [113]. A vaccine containing both $\mathrm{MSP}_{42} 3 \mathrm{D} 7$ and FVO alleles fused together with conserved regions of MSP1 is currently being tested [116].

\subsubsection{Merozoite Surface Protein 2 (MSP2)}

MSP2, a 45-52 kDa glycoprotein is tethered to the membrane a glycosylphosphatidylinosital (GPI) anchor and is the second most abundant protein (based on copy numbers) on the merozoite surface [49]. The protein consists of highly conserved $\mathrm{N}$ - and C-terminal ends flanking a highly polymorphic central repeat region. MSP2 sequences fall into two distinct allelic families namely FC27 and 3D7 (IC-1) [117-120]. Within these allele-families, the central repeats vary in length, number and sequence among isolates. Allele-specific and length polymorphism in MSP2 has been used as the basis for high-resolution genotyping of $P$. falciparum isolates [121, 122]. 
MSP2 is a target of naturally acquired antibodies [123, 124] and antibodies are associated with protection against clinical malaria in some studies [125-128]. Longitudinal studies have suggested allele-specific antibody responses, with encountered strains not being observed in subsequent infections $[129,130]$, while others have shown that individuals can be re-infected with homologous strains [131]. The Combination B vaccine, which was composed of the 3D7 alleles for MSP1, MSP2 and ring-associated erythrocyte surface antigen (RESA) was tested in Phase II trials in Papua New Guinea in the early 1990's. This vaccine, which is discussed in more detail later, was shown to be efficacious, reducing parasite densities significantly with most of the activity attributed to MSP2 [6]. A combination vaccine, MSP1-C1 containing both the 3D7 and FC27 alleles has recently been tested but showed unacceptable reactogenicity due to the adjuvant used and the trial was terminated [132].

\subsubsection{Merozoite Surface Protein 3}

MSP3, also known as Secreted Polymorphic Antigen associated with Merozoites (SPAM) is associated with the merozoite surface. It is secreted into the parasitophorous vacuole of the mature parasite (schizont) where it undergoes proteolytic cleavage [133-135]. Upon rupture of the infected erythrocyte, some MSP3 protein remains associated with the merozoite [133]. MSP3 contains three blocks of a polymorphic heptad repeat flanked by conserved sequences [133]. While initial studies reported limited polymorphism [134, 136] analysis of several isolates has revealed that the sequence is primarily dimorphic in nature with both point mutations and repeat variations in the $\mathrm{N}$-terminal half of the protein with different alleles demonstrating variable antibody binding activity [137]. Antibodies produced in mice can inhibit parasite growth in vitro in co-operation with monocytes [135] but are allele-specific [138]. Furthermore, antibodies to different alleles in endemic human populations are individually associated with protection against clinical episodes [138]. Sequence data from natural parasite populations has confirmed that this antigen is under strong balancing selection and therefore a natural immune target [138].

A MSP3 vaccine based on a long synthetic peptide (MSP3-LSP) covering a relatively conserved region (amino acids 181-276) from strain FC27 has been tested in Phase I trials in Switzerland and later in Burkina Faso and shown to be safe and immunogenic with a strong cytophilic response $[45,139]$. Although the second trial was not designed specifically to test efficacy the number of clinical episodes was measured as a means to monitor safety. Comparison of two MSP3-LSP vaccinated groups (different adjuvants) compared to individuals that received the alternative vaccine (Hepatitis B) demonstrated that the incidence of clinical malaria was three to four-fold lower in the MSP3-LSP vaccine groups [45]. Interestingly however, the degree of protection wanes substantially during the follow up period of 60 days, suggesting that protection may be short-lived. The GMZ2 vaccine comprising a fusion protein containing conserved regions of MSP3 and Glutamate Rich Protein (GLURP) has also been confirmed as safe and immunogenic in Phase I trials [140] and Phase II trials are currently underway [51].

\subsubsection{5kDa Erythrocyte Binding Antigen (EBA175)}

The $175 \mathrm{kDa}$ Erythrocyte Binding Antigen, EBA175, is found in the micronemes, which are located at the apical end of the merozoite [141, 142]. It is a parasite ligand that directly associates with its receptor, Glycophorin A on the surface of uninfected erythrocytes. This 
occurs via an interaction between sialic acids and the Glycophorin A backbone $[143,144]$. The cysteine-rich binding region (RII) is a 616 amino acid region consisting of two regions - F1 and F2, which are known as Duffy binding-like (DBL) domains named so as they are homologous to $P$. vivax Duffy binding protein, DBP. These domains are found in several other adhesion ligands of $P$. falciparum including Erythrocyte Membrane Protein 1 (PfEMP1). Antibodies to EBA175-RII can be induced by immunization in animal models by recombinant EBA175 protein [143] and are acquired in humans naturally exposed to malaria [36, 145-147] and these antibodies can inhibit parasite invasion in vitro $[142,148]$. Studies have suggested that EBA175 alleles are maintained by immune selection [149] and high levels of haplotype diversity are present, most likely as a result of recombination [8]. However, because P. falciparum parasites can vary the use of EBA175 to evade antibodies [150], EBA175 could not be used alone but would need to be combined with other merozoite antigens.

Vaccination of primates with the EBA175 vaccine candidate, EBA175-RII-NG, which is based on the 3D7 allele of RII, resulted in a significant decrease in parasite density after homologous challenge [151]. Clinical trials have proven the vaccines safety and shown that it produces antibody responses in vaccinated individuals. Furthermore, the serum of vaccinated individuals was shown to inhibit the binding of recombinant EBA175-RII to erythrocytes [152].

\subsubsection{Pfs25 and Pfs45/48}

The transmission blocking candidates Pfs 25 and Pfs45/48 are found in the zygote/ookinte and gametocyte stages respectively. These antigens, which migrate as single and double bands respectively, are targets of antibodies that have been shown to block transmission of P. falciparum to the mosquito vector [153-156]. Pfs25 was cloned well before Pfs48/45 [157, 158] and therefore is the only candidate for which a vaccine trial has been carried out. Preclinical development studies are underway for the latter antigen. The attraction of Pfs25 as a vaccine candidate is that it is not expressed in the human host and has relatively limited polymorphism $[159,160]$. It is therefore unlikely to be under the same immune pressures as other antigens, however one downside of this is that natural boosting may not occur unless long lived T-cell responses are elicited by the vaccine. Pfs $48 / 45$ gene knock out experiments demonstrate a key role in male fertility [161]. Anti-Pfs48/45 antibodies in individuals naturally exposed to malaria are associated with transmission blocking activity [162] and given that it is expressed within the human host it is likely to allow natural boosting of antibody responses. Pfs 25 is relatively conserved [163] while Pfs $48 / 45$ shows high levels of diversity worldwide with evidence of diversifying selection and strong geographic structuring $[8,164,165]$.

The only vaccine trial of these two antigens that has been conducted is a phase I trial of the Pfs25 vaccine candidate. However it was halted due to unexpected adverse effects [166]. Preclinical studies have demonstrated a significant increase in the immune response of animal models, and immune-sera had a significant transmission blocking effect [167]. Further clinical trials are planned [51].

\subsubsection{Other Plasmodium falciparum vaccine candidates}

There are several other malaria vaccine candidates currently under development which are based on well-known antigens, such as GLURP, which as mentioned above is being tested in 
combination with MSP3 [140], Liver Stage Antigen 1 (LSA1) being developed as a component of the ME-TRAP vaccine [168] and as a single component vaccine [169]; and MSP4 [170]. More recently discovered antigens demonstrate significant potential as vaccine candidates including members of the Rh family of proteins (Rh1, 2a, 2b, 4 and 5) [171, 172] and the Rh-interacting protein, RIPr [173], Serine repeat antigen 5 (SERA5, reviewed in [174]); MSP6 [175], MSP7 [176] and the pregnancy-associated malaria vaccine candidate, var2csa [177-179]. For some of these antigens, diversity is a significant issue that needs to be evaluated.

Combinations of antigens from different $P$. falciparum lifecycle stages have also been tested. Trials for Spf66, a synthetic combination of peptides from including CSP, MSP1 and two others of unknown origin have now been halted after it was shown to have no effect on malaria [180], NMRC-M3V-Ad-PfCA which is a combination of CSP and AMA1 and is currently in phase 2 trials in the USA [51], GMZ2 which was mentioned above (MSP3 and GLURP, [140]) and the EBA/Rh vaccine candidate, which consists of EBA175 (RIII-V) and $\mathrm{Rh} 2 \mathrm{a} / \mathrm{b}, \mathrm{Rh} 5$ and RIPr binding regions [181]. This vaccine candidate is still undergoing preclinical testing and aims to target different invasion pathways used by the merozoite [172].

\subsection{Plasmodium vivax}

The increasing recognition of the importance of Plasmodium vivax, both as a significant cause of severe malaria and as a major obstacle to malaria control and elimination has exposed some major gaps in our knowledge of this parasite [182]. The vaccine development pipeline is lagging well behind that of P.falciparum and while work is progressing on vaccines targeted at the P.vivax Duffy-binding protein (DBP, [183]), which has long had a human genetic correlate (Duffy negative individuals have a greatly reduced risk of infection) [184], relatively little has been done on the blood-stage antigens homologous to those being intensively tested for P.falciparum [39]. Underlying this lack of development is an urgent need to understand more about the biology of the transmission of the parasite, the extent of its diversity at a population level, and the mechanism of acquiring immunity to it.

One major obstacle impeding P.vivax research is the fact that presently, P.vivax cannot be maintained in long-term culture or at high parasitaemias. In addition, infected individuals typically present with low parasitemia and therefore parasite material is less available than for P. falciparum [185]. Recombinant P.vivax proteins for use in immunoepidemiological studies and vaccine development therefore are often isolated from reference strains, such as Sal1 (Salvador).

Many $P$. vivax vaccine candidates currently being investigated are orthologues of $P$. falciparum vaccine candidate antigens [38, 39]. However, these two species have distinct biological features, the most obvious being the ability of $P$. vivax to form dormant liver stages, and also their variable transmission in different regions across the globe. Therefore it is difficult to base conclusions for P.vivax, on what is known for P.falciparum. Many experts believe that a malaria vaccine will need to contain a combination of both $P$. falciparum and $P$. vivax antigens to be globally effective, since many regions of the world are burdened with both species [38]. 
Only two P.vivax vaccine candidates, the circumsporozoite surface protein (CSP) and the gametocyte antigen, Pvs25, have been tested in clinical trials to date [38]. However, a host of additional P.vivax proteins are currently under investigation as potential vaccine candidates, including PvDBP [38], PvTRAP [186], PvMSP-1 [187], PvAMA-1 [188]; and the transmission blocking candidate, Pvs28 [38]. Additional antigens identified as potential P.vivax vaccine candidates include PvMSP3, PvMSP4, PvMSP5 and PvRBPs I and II [38]. PvMSP9 has also been revealed as a promising vaccine candidate in recent studies [189].

\section{Variant-specific immunity}

Naturally acquired immunity to malaria develops only after years of exposure to infection by Plasmodium parasites. The extensive genetic diversity that is characteristic of malaria surface antigens provides one explanation for this, especially because immune responses are variant-specific. Eventually, antibodies to all of the variants in the parasite population are acquired or reach a threshold whereby protection against clinical episodes is achieved (reviewed in [190]).

\subsection{Identifying targets of human immunity}

Numerous epidemiological studies have investigated the role of malarial antigens as natural targets of human immunity with conflicting estimates of their protective effect. While differences in study methodology, transmission intensity, and the rate of natural immunity acquisition [190, 191] will account for some of the discrepancies, parasite genetic diversity is likely to play a major role. This is because the prevalence of the major allelic types of specific antigens and subsequent acquisition of allele-specific immunity varies across populations.

A recent systematic review and meta-analysis investigating the protective effect of antibody responses to merozoite antigens, highlighted the issues of genetic diversity in immunoepidemiological studies [192]. This review pooled all the published evidence for the association between anti-merozoite antibodies and the incidence of P. falciparum for each antigenic allele. For each allele, individual study estimates often showed large degrees of heterogeneity and comparing pooled estimates across alleles for the same antigen either showed similar (e.g. MSP1, MSP2) or very different (e.g. MSP1 19 , MSP3, AMA1, GLURP) magnitudes of a protective effect.

A major contributor to the heterogeneity in protective estimates is the fact that allele-specific antibody response to the strain causing the malaria episode was not measured in these studies. If antibody-mediated protection is largely allele-specific then the true causal protective effect will be underestimated in studies that do not use allele-specific $P$. falciparum outcomes. For example, meta-analysis of studies investigating the protective effects of anti$\mathrm{MSP}_{3 \mathrm{D} 7}$ and $\mathrm{MSP}_{\mathrm{FC} 27}$ responses showed no evidence of a reduced risk of symptomatic $P$. falciparum (all strains combined) [192]. Four studies included allele-specific endpoints; two studies in PNG showed protective MSP2 responses to homologous strains [125, 128] whereas studies in South America and Africa [193, 194], show no evidence of a protective effect of pre-existing MSP2 allele-specific immunity on clinical episodes with homologous parasites. 
It is clear that the importance of allele-specific immunity to malaria has been understudied in sero-epidemiological studies. There is a real need to incorporate strain-specific responses and endpoints in immuno-epidemiological studies to address whether protective responses against particular antigens are strain-transcending or strain-specific. This research is imperative so we can elucidate whether sub-unit vaccines including only one allelic type might protect against a small subset of the parasite population and thus select for parasites bearing heterologous alleles.

\subsection{Vaccine trials and antigen polymorphism}

Significant sequence polymorphism is present in most of the vaccine antigens that are currently in clinical trials, as well as in many of the lead candidate antigens that are in preclinical development. This includes P. falciparum antigens such as CSP (RTS,S vaccine), MSP1-42, MSP2, MSP3, AMA1, and P. vivax antigens, such as DBP [36]. Presently, there is only a very limited understanding of how sequence polymorphisms relate to antigenic diversity or the potential for polymorphisms to mediate vaccine escape.

Different approaches can be taken to overcome antigenic diversity present in vaccine candidates. One approach is to include multiple alleles, or variants, of an antigen such that the vaccine-induced responses cover most of the different alleles circulating in a population; referred to as multivalent vaccines. This approach has been widely used in the development of vaccines for viral and bacterial pathogens. Examples include vaccines for Streptococcus pneumonia, Neisseria meningitidis, and influenza. Among malaria vaccines, multivalent vaccines are in development for several antigens including MSP2 [132] and AMA1 [195] of $P$. falciparum. The level of polymorphism seen among different candidate antigens varies substantially, being high for some candidate antigens, such as AMA1, compared to others, such as EBA175 [8]. Antigens that have a lower level of diversity may be more attractive candidates for vaccine development; however, multiple factors need to be considered in prioritising the many potential antigens for development [36]. An alternative approach is to design vaccine constructs that include a conserved region of the antigen so that vaccineinduced responses would target epitopes common to all variants of the antigen. This approach is being taken for $P$. falciparum MSP3, whereby the vaccine antigen includes a conserved stretch of sequence (known as MSP3-LSP) towards the C-terminal end of MSP3 and reportedly omits the highly polymorphic epitopes [196]. Interestingly however, recent population genetic surveys have shown that T-cell epitopes within MSP3-LSP are somewhat polymorphic [138].

A well-known example of the importance of antigenic diversity in vaccine development is the phase I/IIb trial of the Combination B vaccine for P. falciparum malaria [6], which was mentioned earlier. The vaccine had significant efficacy in reducing parasite densities during follow-up and the anti-malarial effect of the vaccine is thought to be mediated primarily by responses to MSP2, rather than other components of the vaccine. Although MSP2 is polymorphic, nearly all sequences can be grouped into two allelic families, 3D7 and FC27. The Combination B vaccine contained only the 3D7 form of MSP2 and, consequently, infections in vaccinated individuals were over-represented by FC27 allelic types. This implies that the vaccine only had substantial efficacy against infections containing the vaccine allele, 3D7. To address this, an MSP2 vaccine containing both of the 
major allelic types is in development [132]. A more recent example of the challenge of antigenic diversity in malaria vaccines is AMA1. A phase II trial of a vaccine containing a single allele of $P$. falciparum AMA1 (3D7-allele) had no significant clinical efficacy when all malaria episodes were considered [46]. However, as was mentioned earlier, the vaccine demonstrated $68 \%$ efficacy against malaria episodes caused by AMA1 alleles that were similar in sequence to the vaccine allele. The development of approaches to overcome diversity in AMA1 and generate responses that give broad population coverage are ongoing.

The most advanced vaccine in development, RTS,S, is based on a region of $P$. falciparum CSP [25]. Because CSP is polymorphic, an obvious question was whether diversity in CSP may explain the partial efficacy of the RTS,S vaccine. Analyses of infecting genotypes in one phase II trial of RTS,S suggested that this was not the case as there was no evidence of selection for infections containing non-vaccine genotypes among vaccinated versus control subjects [197, 198]. These findings highlight that although antigen polymorphism needs to be considered in vaccine development, it may not be a major issue for all vaccines. Clearly, there is a lot more we need to understand about the nature and specificity of responses and the importance of sequence diversity.

There are a number of other promising antigens in pre-clinical development or early clinical trials where issues of polymorphisms have been explored. The leading P. vivax vaccine candidate, DBP [199] has significant polymorphism that appears to impact on the efficacy of inhibitory antibodies [200], and protective immunity shows a degree of strainspecificity [201]. The erythrocyte binding region of the $P$. falciparum vaccine candidate EBA175, also has significant polymorphism [149, 202]. Interestingly, studies suggest that these polymorphisms have little impact on the efficacy of vaccine-induced inhibitory antibodies in vitro $[203,204]$. However, the ability of $P$. falciparum to vary the expression and use of EBA175 during invasion may be an important means of immune evasion [150], which presents different challenges in vaccine design. Another example is the PfRh invasion ligand family. One member that appears to be an important target of acquired immunity, PfRh2, has significant polymorphism in the N-terminal region [171], but studies suggest it is possible to target a C-terminal conserved stretch of the protein in vaccine design [172]. The challenge of addressing diversity in vaccine development is greatest for PfEMP1, which is expressed on the surface of infected RBCs. PfEMP1 shows extreme sequence diversity (e.g. $[205,206])$ and is encoded by a family of around 60 var genes per genome that enables clonal antigenic variation through expression of different PfEMP1 variants [207]. A vaccine trial in non-human primates showed strain-specific protective efficacy [208] and it is not clear how this extensive antigenic diversity can be overcome. However, one specific PfEMP1 variant, known as var2csa, plays a key role in placental infection [209] and may be a suitable target for a vaccine that helps protect pregnant women from the complications of malaria during pregnancy. Although this single variant has a high level of polymorphism [210], recent studies suggest the extent of antigenic diversity may not be high as there appear to be many shared epitopes that are common to different variants [211, 212], and antibodies that cross-react to different variants have been induced by vaccination $[213,214]$ and are acquired through natural exposure [211, 215] 


\section{How can population genetics inform malaria vaccine development?}

Population genetics is the study of allele frequency distributions and changes that occur in response to the four major forces of evolution: natural selection, genetic drift, mutation and gene flow [216]. All of the current malaria vaccine candidates are potential targets of positive balancing selection due to pressure from human immune responses. Balancing selection maintains alleles at low to medium frequencies and therefore no single allele is likely to provide broad protection [217]. Population genetic analyses can reveal insight into the extent and distribution of alleles and has been important in highlighting antigens as targets of natural immunity [218, 219].

Scores of population genetic surveys have been conducted on malaria vaccine candidates, including isolates from countries in every major malaria-endemic corner of the world. However, the data has generally not been used in the formulation of malaria candidate vaccines. Vaccine developers have included alleles isolated from wellcharacterised reference strains, 3D7 (and its parent NF54, origin unknown), FVO (Vietnam) or FC27 (Papua New Guinea). However, amongst the substantial sequence data available for many countries for several leading malaria vaccine antigens, these alleles are either completely absent or found at low frequencies among naturally circulating parasites (Figure 1, [8]).

A recent metapopulation genetic analysis has summarised the known diversity of twelve leading malaria vaccine candidates [8]. After compiling all available published population data on malaria vaccine candidates, either currently in vaccine trials or in preclinical development, a database of almost 5000 sequences was used to investigate the range and distribution of diversity. Only non-synonymous polymorphisms were investigated as synonymous polymorphisms do not change the protein structure and are therefore antigenically irrelevant. Table 1 summarises the results observed for the ten antigens analysed in this study, as well as other $P$. falciparum and $P$. vivax antigens that are leading malaria vaccine candidates.

The data presented in Table 1 demonstrates that the majority of current malaria vaccine candidate antigens have many distinct haplotypes and this emphasises the problem of diversity in developing a broadly effective malaria vaccine [35]. However, without knowledge of this natural diversity it will be difficult to assess vaccine trials to the full extent. In addition, for genes encoding merozoite antigens including AMA1, EBA175 and MSPs 1-4 the full breadth of diversity was present in all populations with no evidence of geographic population structure, suggesting that they are under strong immune selection. However, genes encoding the non-merozoite antigens including CSP, TRAP, LSA1 and Pfs48/45 showed variable levels of diversity, which were related to transmission levels, and there was evidence of geographic population structure [8]. The consequence of this contrasting distribution of diversity for malaria vaccine design is that a diversity-covering vaccine may be possible for merozoite antigens but for the non-merozoite antigens identifying common alleles across all populations will be difficult. Nevertheless, diversity in the non-merozoite antigens does not appear to be primarily structured by immune selection, and therefore may not be as immunologically relevant as that for the merozoite antigens. 


\begin{tabular}{|c|c|c|c|c|c|c|c|}
\hline Antigen & Expression & Domain & Continents & Countries & $\begin{array}{c}\text { Total isolates } \\
\text { sampled } \\
\text { (range) }\end{array}$ & $\begin{array}{c}\text { Total } \\
\text { haplotypes } \\
\text { (range) }\end{array}$ & References \\
\hline \multicolumn{8}{|l|}{$\begin{array}{l}\text { Plasmodium } \\
\text { falciparum }^{a}\end{array}$} \\
\hline CSP & sporozoite & C-terminal & 3 & 13 & $604(9-143)$ & $71(3-20)$ & $\begin{array}{c}\text { reviewed in } \\
{[8]}\end{array}$ \\
\hline TRAP & sporozoite & N-terminal & 2 & 3 & $100(8-48)$ & $84(8-37)$ & $\begin{array}{c}\text { reviewed in } \\
{[8]}\end{array}$ \\
\hline LSA1 & liver stage & N-terminal & 3 & 4 & $74(10-22)$ & $13(3-7)$ & $\begin{array}{c}\text { reviewed in } \\
{[8]}\end{array}$ \\
\hline GLURP & $\begin{array}{l}\text { sporozoite/ } \\
\text { gametocyte }\end{array}$ & Region 0 & 3 & 3 & $48(9-11)$ & $22(2-9)$ & $\begin{array}{c}\text { reviewed in } \\
{[8]}\end{array}$ \\
\hline AMA1 & merozoite & Domain I & 3 & 11 & $572(8-162)$ & $181(6-68)$ & $\begin{array}{c}\text { reviewed in } \\
{[8]}\end{array}$ \\
\hline EBA175 & merozoite & Region II & 2 & 3 & $135(30-48)$ & $51(15-23)$ & $\begin{array}{c}\text { reviewed in } \\
{[8]}\end{array}$ \\
\hline MSP1 & merozoite & MSP119 & 3 & 11 & $\begin{array}{c}2237(18- \\
1368)\end{array}$ & $20(1-15)$ & $\begin{array}{c}\text { reviewed in } \\
{[8]}\end{array}$ \\
\hline MSP2 & merozoite & $\begin{array}{c}\text { Blocks } 2 \text { and } \\
3\end{array}$ & 2 & 3 & 392 (n.d) & 275 (n.r.) & $\begin{array}{c}\text { reviewed in } \\
{[8]}\end{array}$ \\
\hline MSP3 & merozoite & $\begin{array}{l}\text { Repeat } \\
\text { region }\end{array}$ & 2 & 2 & $124(75-86)$ & $21(9-12)$ & $\begin{array}{c}\text { reviewed in } \\
{[8]}\end{array}$ \\
\hline MSP4 & merozoite & Full length & 2 & 4 & $142(12-42)$ & $47(9-23)$ & $\begin{array}{c}\text { reviewed in } \\
{[8]}\end{array}$ \\
\hline MSP3/6 & merozoite & & 1 & 2 & $117(51-66)$ & n.r. & {$[218,219]$} \\
\hline Rh2 & merozoite & $\begin{array}{l}\text { Binding } \\
\text { region }\end{array}$ & 1 & 1 & $33(15)$ & n.r. (13) & [171] \\
\hline Rh4 & merozoite & $\begin{array}{l}\text { Binding } \\
\text { region }\end{array}$ & 1 & 1 & $23(12)$ & $9(4)$ & $\begin{array}{c}\text { Reiling et al. } \\
\text { unpublished } \\
\text { data }\end{array}$ \\
\hline Pfs $48 / 45$ & gametocyte & Full length & 3 & 4 & $55(9-15)$ & $19(2-8)$ & $\begin{array}{c}\text { reviewed in } \\
{[8]}\end{array}$ \\
\hline Pfs28 & ookinete & \multicolumn{6}{|c|}{ no population data available } \\
\hline var $2 \operatorname{cs} a$ & trophozoite & DBL3 & 2 & 3 & $124(15-54)$ & n.r. & [211] \\
\hline $\begin{array}{c}\text { Plasmodium } \\
\text { vivax }^{b}\end{array}$ & & & & & & & \\
\hline CSP & pre-eryth & $\begin{array}{l}\text { Central } \\
\text { repeat }\end{array}$ & 2 & 2 & $168(31-137)$ & n.r. $(13-25)$ & {$[220,221]$} \\
\hline TRAP & pre-eryth & $\begin{array}{c}\text { Regions II- } \\
\text { IV }\end{array}$ & 2 & 2 & $74(37-37)$ & 54 (n.r.) & [222] \\
\hline AMA1 & merozoite & Full length ${ }^{c}$ & 2 & 2 & $96(23-73)$ & n.r. $(15-18)$ & [223] [224] \\
\hline MSP1 & merozoite & Full length ${ }^{\mathrm{c}}$ & 2 & 7 & $196(40-116)$ & n.r. $(12-31)$ & {$[187,225]$} \\
\hline DBP & merozoite & Region II & 2 & 8 & $675(11-123)$ & n.r. (9-73) & [226] \\
\hline Pvs28 & ookinete & All & 1 & 1 & 20 & 2 & [227] \\
\hline Pvs25 & ookinete & All & 1 & 1 & 30 & 4 & [227] \\
\hline
\end{tabular}

a.Total includes both natural populations and other isolates, range includes only natural populations; $b$. Total (range) includes only natural populations; c.Individual domains also analysed, data not shown; n.r. result not available.

Table 1. Summary of population genetic data for leading malaria vaccine candidates 


\subsection{Sampling diversity}

An important consideration when investigating the genetic diversity of a vaccine candidate is the origin and number of samples required to obtain reliable allele frequency estimates. In early studies, only a handful of parasite isolates from diverse geographic origins were used to investigate diversity, however more recently a number of investigations on larger numbers of locally circulating field isolates which can represent natural parasite populations, have been completed (reviewed in [8], Table 1). While a geographically disparate sampling approach can provide insights into levels of polymorphism, immune selection and can allow the extent of diversity to be predicted, it cannot provide reliable information on allele frequencies. The latter approach is more appropriate if data will be used for prioritizing common alleles for vaccine development. However it is critical that large sample sizes of a minimum of 30-50 isolates be used to obtain a reliable estimate of diversity and values approaching natural allele frequencies. Once defined, natural allele frequencies can provide an indication of the minimum proportion of the parasite population that would be covered by a particular vaccine formulation. Further analysis, discussed below, can identify relationships amongst alleles and therefore the potential for crossreactivity between distinct alleles.

\subsection{Defining the extent of diversity}

In population genetics, the extent of diversity at a defined locus is measured using a number of different statistics. These include statistics that are simple to estimate: e.g. the number of alleles or haplotypes and more complex statistics such as the allelic richness, which is normalised for sample size [228] and therefore is useful to compare among populations if samples sizes vary considerably. Other statistics include the nucleotide diversity $(\pi)$, which is the average proportion of sites that are polymorphic within a group of sequences; the average number of differences and the expected heterozygosity, all of which can be easily calculated with the help of a myriad of population genetic software (reviewed in [229]). The most informative statistics for vaccine design includes the numbers of alleles or haplotypes that need to be considered in developing a broadly efficacious malaria vaccine. For example if we consider the data in Table 1, some antigens have larger numbers of haplotypes than others, such as CSP with 71 haplotypes while MSP3 has only 21 even though the two datasets were similar in size, showing that CSP is the more diverse antigen [8].

\subsection{Defining the distribution of diversity}

\subsubsection{Allele and haplotype frequencies}

Knowledge of the distribution of alleles and haplotypes (variants) is critical both for vaccine design and for monitoring the effects of vaccine trials. In trials for vaccine candidate antigens with allele-specific immunity, a high frequency variant has a higher likelihood of resulting in a protective effect than a low frequency variant (if the vaccine construct covers polymorphic regions). Furthermore, the identification of geographically distinct population structures is an indication that variants may be present at different frequencies. In this situation, it is possible that a vaccine based on a common allele in one population that is rare in another population, may have differential effects across the two populations. Similarly, if 
a vaccine is based on a variant that is found at low frequencies at a testing site, the positive effects of variant-specific immunity may be dampened by infection with parasites carrying non-vaccine alleles. The vaccine may then be interpreted as being non-protective, unless variant-specific end-points are included by genotyping post-vaccine infections. Another important consideration in this scenario is that the statistical power to measure variantspecific efficacy will be limited with only a small number of infections carrying the vaccine allele.

The importance of allele/haplotype frequencies in vaccine design has been demonstrated by a study that measured $\mathrm{MSP}_{19}$ diversity at a vaccine-testing site in Mali. High throughput genotyping of six common $\mathrm{MSP}_{19}$ polymorphisms in more than 2000 isolates showed that there were two highly prevalent haplotypes (FVO, 46\% and FUP, 36\%) whereas the majority of the haplotypes were relatively rare $(<10 \%)$. The common haplotypes remained common over long periods of time ( $>44 \%$ and $34 \%$ respectively), with fluctuations that could be explained by frequency dependant selection [113]. The vaccine haplotype, 3D7, was the third most common, being found at a frequency of $16 \%$ (14-18\%) throughout the study period. The authors concluded that the previous $\mathrm{MSP}_{19}$ vaccine trial [114] probably failed as a result of a lack of parasites harbouring $3 \mathrm{D} 7 \mathrm{MSP} 1_{19}$ haplotypes. Studies have investigated changes in vaccine antigen haplotype frequencies over time under natural conditions $([93,230,231])$ but there have been e.g. relatively few during vaccine trials $[98,115]$. More studies are needed to monitor fluctuations that occur in parasite populations over time, especially under the influence of natural immune or vaccine-mediated selection.

\subsubsection{Clustering patterns}

Cluster analyses have been used to identify population substructure within a given sample to understand the underlying population biology of $P$. falciparum [232]. This analysis has also been adapted to understand relationships among haplotypes of several malaria vaccine candidates [8, 91, 92, 226]. A study of 150 AMA1 sequences by Xin Zhuan Su and colleagues demonstrated that they clustered into six distinct subgroups. Some evidence was also presented that sera from rabbits immunized with AMA1 variants from one cluster tended to inhibit invasion of parasite isolates carrying sequences from the same cluster, but were less active against those from other clusters, suggesting that different clusters contain immunologically distinct sequences [91]. Later studies, including 506 AMA1 domain I sequences analysed have suggested up to 16 clusters [92]. Although the full utility of clustering patterns is yet to be confirmed, they may be used as a guide to select representative variants to cover diversity as well as to predict the effects of a vaccine trial within a defined geographic area. This is particularly important because different malaria antigens show different clustering patterns ([8], Figure 2). If clusters represent immunologically distinct subgroups, the patterns observed in Figure 2 suggest that vaccination with CSP would be significantly more effective in Africa and parts of the Americas, whereas for MSP1 the effects would be similar among many populations. Different polymorphisms have varying immunological significance $[85,97]$ and a better understanding of the relationship between polymorphisms and antigenic diversity will help advance the development of clustering algorithms. 
A. CSP

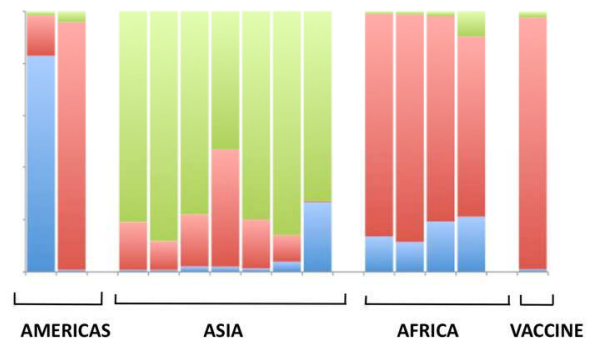

B. MSP1

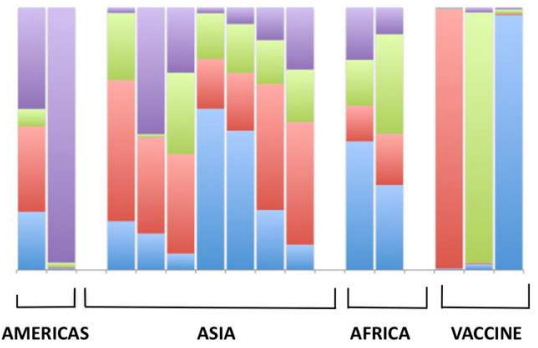

Fig. 2. Cluster analysis of sequences for (A) the pre-erythrocytic antigen, CSP and (B) the merozoite antigen, $\mathrm{MSP}_{19}$. Non-synonymous haplotypes were submitted to cluster analysis using STRUCTURE software [233]. Each bar represents the mean membership co-efficient for the parasite population of each country and colours represent the mean membership to each of the clusters. Vaccine alleles include CSP: 3D7; MSP1 19: 3D7, FVO or FC27. Figure adapted from [8].

\subsubsection{Networks}

Network analysis originated as a mathematical tool to understand social relationships and has been used to study the transmission of infectious diseases [234]. In population genetics, it has been adapted to explore relationships among sequences by linking haplotypes that are identical at a predefined proportion of polymorphic sites [8, 235]. As each haplotype (node) may have multiple connections (edges), this analysis has the potential to define not only distinct clusters or subgroups of highly related sequences but also the relationships among them. Furthermore, it can identify the location of less frequently observed admixed haplotypes in the network, which may represent novel recombinants. Barry et al. [8] have explored the distribution of haplotypes using network analysis and found that the network was concordant with clustering patterns for ten leading malaria vaccine antigens (Figure 3 shows the results for AMA1 as an example). In addition, they demonstrated that haplotypes

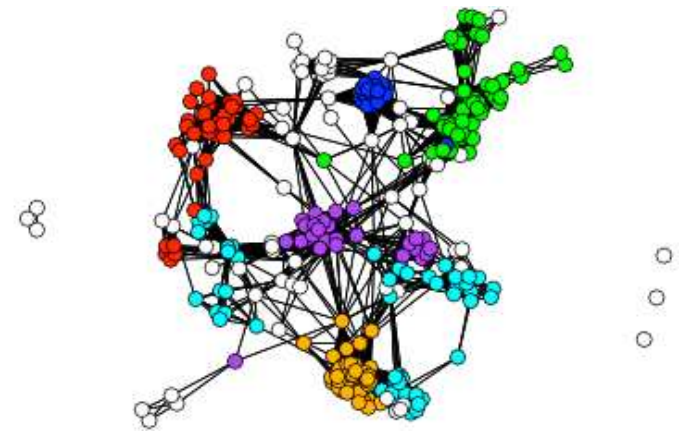

Fig. 3. Network of AMA1 (domain I) sequences. Each node (circle) represents a haplotype, shaded in colour to highlight cluster-membership or white for admixed haplotypes (as defined by the Structure analysis discussed above [233]). Nodes are tied by edges (black lines) demonstrating that they share a predefined threshold of 48 nsSNPs. Admixed haplotypes originating from isolates with unknown origin are shaded in white (unless they were vaccine haplotypes). Adapted from [8]. 
that were identified as "admixed" in the cluster analysis (i.e. $<75 \%$ of sequences assigned into any one cluster), often formed connections between one or more lobes of the network suggesting that these represent novel recombinants resulting from exchange between sequences from the linked clusters. Such recombinants might allow evasion of naturally acquired- or vaccine-mediated immune responses if vaccine formulations were comprised only of haplotypes from distinct subgroups or clusters. Network analysis will allow shifts in the proportion of haplotypes within each cluster and admixed/recombinant haplotypes to be monitored during vaccine trials.

\subsection{Immune selection}

Population genetic analyses can identify signatures of balancing selection in loci that are targeted by natural immune responses and therefore allow vaccine candidates to be ranked $[66,218,219,236]$. While data from geographically diverse isolates can be useful, the ability to identify balancing selection is strengthened by allele frequency data from natural parasite populations [237]. Comparative studies, investigating polymorphism and allele frequencies by deep population sampling of several novel vaccine candidates have been done $[218,219$, $224,237,238]$. These have demonstrated the relative levels of balancing selection and therefore whether particular candidates are stronger immune targets than others, but may also partially reflect the tolerance of a particular antigen to high levels of mutation.

Balancing selection can be measured using a variety of different statistics, however the Hudson Kreitman Aguade ratio (HKAr), which is determined by calculating levels of divergence between species and dividing by the amount of diversity within a species [239] has been shown to be most informative for assessing selection in any dataset, including small numbers of isolates from diverse geographic locations. However, this also relies on the availability of sequences from the most closely related species for which genome data is available, namely Plasmodium reichenowi. Additional $P$. reichenowi isolates have been collected by researchers and may also be used to obtain further sequence data to increase the reliability of this statistic [240]. Another statistic that has proven to be reliable for predicting balancing selection, is Tajima's D, which identifies departures from neutrality by measuring the number of polymorphic sites $(S)$ in relation to the nucleotide diversity $(\pi)$ [241]. Tajima's $\mathrm{D}$ requires allele frequency data and therefore can only be effectively used on population samples, with large sample sizes (>50 isolates) being the most informative [218].

\subsection{Credentialing polymorphisms}

It is clear that some antigens such as AMA1 and CSP have many polymorphic sites, while others such as EBA175 and MSP4 remain relatively conserved [8]. However, this is probably more a reflection of functional constraints or degree of immune exposure, than indicating their capacity to be an effective vaccine candidate. The challenge is how to determine which polymorphisms will be critical to vaccine design. Investigators have used different approaches to identify antigenically relevant polymorphisms, including three-dimensional structural modelling, immunological assays and mutational analysis [91, 97]. However, population genetic data - which may be easier to collect - can be highly informative.

When choosing which polymorphisms to consider for vaccine design it is important to define the allele frequencies for each polymorphic site. If the minor allele frequency (MAF) 
is $<10 \%$, at least $90 \%$ of isolates will carry the predominant allele, and therefore a vaccine candidate carrying the major allele will target the majority of the parasite population. Mapping regions of significant balancing selection (where allele frequencies will be lowmedium) will identify regions that have accumulated polymorphisms as a result of being directly targeted by the immune response [236]. These analyses can help identify regions that are targets of functional immune responses. The analysis can be done by sliding window analyses of Tajima's D or measuring the HKAr for different protein domains. As balancing selection maintains balanced allele frequencies across different populations, it is also possible to use the interpopulation differentiation statistic, Wrights $\mathrm{F}_{\mathrm{ST}}$ to measure differences in allele frequencies between populations. A low and insignificant Wrights $\mathrm{F}_{\mathrm{ST}}$ value will demonstrate that allele frequencies are similar among populations and polymorphic regions potentially associated with immune escape [236, 242]. An additional important approach to determine key polymorphisms is by monitoring longitudinal infection dynamics in cohort studies, which may include vaccine trials [92]. If specific polymorphisms allow immune escape, then subsequent infections will carry alternative alleles to those of previous infections. Similarly, in vaccine trials, post-vaccination infections can be monitored for the presence of vaccine alleles. Antigenicity can then be determined by comparing allele frequencies in the vaccinated and control groups.

\section{Conclusion: The way forward}

The malaria vaccine development pipeline has suffered many setbacks and complications over the decades since the first malaria vaccine was trialed. Nevertheless, it is our opinion that with carefully planned vaccine trials and an understanding of naturally circulating parasite diversity, made possible through technological advances in next generation sequencing and dedicated bioinformatics efforts, a broadly effective subunit malaria vaccine is achievable. With respect to developing such a vaccine we put forward the following recommendations for the design and testing of the next generation of malaria vaccines:

iv. Prioritise vaccine candidates: Many malaria vaccine candidate antigens have reached vaccine trials simply because they were discovered first. Genome sequencing and "omic" technologies have now identified several antigens that may have greater potential to induce protective immunity to a wide variety of parasite strains than these more traditional candidates. As the pipeline to clinical trials and eventual licensing is an arduous and vastly expensive journey, a thorough review of all candidates including those recently discovered and also in development needs to be done to identify those with the greatest potential to elicit protective immunity against malaria.

v. Prioritise vaccine alleles: Dozens of population genetic studies have highlighted the issue of high levels of genetic diversity in candidate antigens and the low prevalence of the vaccine alleles currently being used, in natural parasite populations. Researchers now have the opportunity to mine already compiled datasets (e.g. [8]) or to rapidly sequence large numbers of field samples in vaccine testing sites to identify high frequency alleles for testing in single or multivalent vaccines. Notably, for a large number of leading malaria vaccine candidates under strong balancing selection, these alleles may be common across populations.

vi. Allele-specific endpoints for vaccine trials: Few malaria vaccine trials have evaluated allele frequencies either before, during, or after vaccine trials have taken place and 
therefore it is not possible to assess whether any allele-specific protection has been induced in individuals receiving the vaccine. Given the relatively low cost (compared to conducting a vaccine trial) and ready availability of high throughput genotyping and NextGen sequencing technologies, this step should certainly be included in future vaccine trials and could even be done in retrospect if samples are still available. However, it is important to note that in some trials where the allele used to formulate the vaccine is found at low frequencies in the testing site, sample size may be too low to evaluate any protective effects.

vii. Parasite population monitoring: Even after an effective vaccine(s) has been developed and rolled out, sustained and intensive surveillance efforts will be essential to monitor population allele frequencies on a regular basis. This will identify significant fluctuations that might impact on vaccine efficacy or newly emerging alleles that may allow antigenic escape. With data obtained through such a surveillance system, formulations may then be adapted $n$ a manner analogous to the Influenza vaccine, to ensure continued protection against malaria.

A broadly effective malaria vaccine continues to be one of the most sought after strategies in the fight against malaria, however the diversity of many of the parasite antigens currently being considered as subunit vaccine candidates is an obstacle that must be overcome. Natural malaria parasite populations are complex and dynamic communities, but modern molecular and analysis technologies are providing new ways to understand them. It is therefore important that researchers consider using population genetics to inform malaria vaccine development from target discovery through to vaccine design and to assess the effects of clinical trials.

\section{Acknowledgments}

The authors are grateful for the support of the National Health and Medical Research Council (NHMRC) of Australia through project grants 488221 and 1003825. JB is supported by a Senior Research Fellowship; JCR by a Principal Research Fellowship; and FJIF by a Training Fellowship from the NHMRC. This work was made possible through Victorian State Government Operational Infrastructure Support and Australian Government NHMRC IRIISS.

\section{References}

[1] Moran, M., Guzman, J., Ropars, A, Jorgensen, M., McDonald, A., Potter, S. and Selassie, H. , The Malaria Product Pipeline: Planning for the future. 2007, The George Institute for International Health: Sydney.

[2] Organization, W.H., Malaria Vaccine Rainbow Table. 2008.

[3] Doolan, D.L., C. Dobano, and J.K. Baird, Acquired immunity to malaria. Clin Microbiol Rev, 2009. 22(1): p. 13-36, Table of Contents.

[4] $\mathrm{Mu}$, J., et al., Genome-wide variation and identification of vaccine targets in the Plasmodium falciparum genome. Nat Genet, 2007. 39(1): p. 126-30.

[5] Fluck, C., et al., Strain-specific humoral response to a polymorphic malaria vaccine. Infect Immun, 2004. 72(11): p. 6300-5. 
[6] Genton, B., et al., A recombinant blood-stage malaria vaccine reduces Plasmodium falciparum density and exerts selective pressure on parasite populations in a phase 1-2b trial in Papua New Guinea. J Infect Dis, 2002. 185(6): p. 820-7.

[7] Takala, S.L. and C.V. Plowe, Genetic diversity and malaria vaccine design, testing and efficacy: preventing and overcoming 'vaccine resistant malaria'. Parasite Immunol, 2009. 31(9): p. 560-73.

[8] Barry, A.E., et al., Contrasting population structures of the genes encoding ten leading vaccinecandidate antigens of the human malaria parasite, Plasmodium falciparum. PLoS One, 2009. 4(12): p. e8497.

[9] Koch, R., Dritter Bericht uber die Thatigkeit der Malaria Expedition. . British Medical Journal, 1900: p. 1183-1186.

[10] McGregor, I.A., The Passive Transfer of Human Malarial Immunity. Am J Trop Med Hyg, 1964. 13: p. SUPPL 237-9.

[11] Nussenzweig, R.S., et al., Protective immunity produced by the injection of $x$-irradiated sporozoites of plasmodium berghei. Nature, 1967. 216(111): p. 160-2.

[12] Clyde, D.F., et al., Immunization of man against sporozite-induced falciparum malaria. Am J Med Sci, 1973. 266(3): p. 169-77.

[13] Clyde, D.F., et al., Specificity of protection of man immunized against sporozoite-induced falciparum malaria. Am J Med Sci, 1973. 266(6): p. 398-403.

[14] Hoffman, S.L., et al., Protection of humans against malaria by immunization with radiationattenuated Plasmodium falciparum sporozoites. J Infect Dis, 2002. 185(8): p. 1155-64.

[15] Yoshida, N., et al., Hybridoma produces protective antibodies directed against the sporozoite stage of malaria parasite. Science, 1980. 207(4426): p. 71-3.

[16] Vanderberg, J.P., M.M. Weiss, and S.R. Mack, In vitro cultivation of the sporogonic stages of Plasmodium: a review. Bull World Health Organ, 1977. 55(2-3): p. 377-92.

[17] Robson, K.J., et al., A highly conserved amino-acid sequence in thrombospondin, properdin and in proteins from sporozoites and blood stages of a human malaria parasite. Nature, 1988. 335(6185): p. 79-82.

[18] Rogers, W.O., et al., Characterization of Plasmodium falciparum sporozoite surface protein 2. Proc Natl Acad Sci U S A, 1992. 89(19): p. 9176-80.

[19] Guerin-Marchand, C., et al., A liver-stage-specific antigen of Plasmodium falciparum characterized by gene cloning. Nature, 1987. 329(6135): p. 164-7.

[20] Holder, A.A., et al., A malaria merozoite surface protein (MSP1)-structure, processing and function. Mem Inst Oswaldo Cruz, 1992. 87 Suppl 3: p. 37-42.

[21] Smythe, J.A., et al., Identification of two integral membrane proteins of Plasmodium falciparum. Proc Natl Acad Sci U S A, 1988. 85(14): p. 5195-9.

[22] Coppel, R.L., et al., Isolate-specific S-antigen of Plasmodium falciparum contains a repeated sequence of eleven amino acids. Nature, 1983. 306(5945): p. 751-6.

[23] Deans, J.A., et al., Vaccination trials in rhesus monkeys with a minor, invariant, Plasmodium knowlesi 66 kD merozoite antigen. Parasite Immunol, 1988. 10(5): p. 535-52.

[24] Hodder, A.N., et al., The disulfide bond structure of Plasmodium apical membrane antigen-1. J Biol Chem, 1996. 271(46): p. 29446-52.

[25] Agnandji, S.T., et al., First results of phase 3 trial of RTS,S/AS01 malaria vaccine in African children. N Engl J Med, 2011. 365(20): p. 1863-75.

[26] Florens, L., et al., A proteomic view of the Plasmodium falciparum life cycle. Nature, 2002. 419(6906): p. 520-6. 
[27] Gardner, M.J., et al., Genome sequence of the human malaria parasite Plasmodium falciparum. Nature, 2002. 419(6906): p. 498-511.

[28] Doolan, D.L., et al., Profiling humoral immune responses to P. falciparum infection with protein microarrays. Proteomics, 2008. 8(22): p. 4680-94.

[29] Doolan, D.L., et al., Utilization of genomic sequence information to develop malaria vaccines. J Exp Biol, 2003. 206(Pt 21): p. 3789-802.

[30] Butler, D., Mosquito production mooted as fast track to malaria vaccine. Nature, 2003. 425(6957): p. 437.

[31] Epstein, J.E., et al., Live attenuated malaria vaccine designed to protect through hepatic CD8 T cell immunity. Science, 2011. 334(6055): p. 475-80.

[32] van Dijk, M.R., et al., Genetically attenuated, P36p-deficient malarial sporozoites induce protective immunity and apoptosis of infected liver cells. Proc Natl Acad Sci U S A, 2005. 102(34): p. 12194-9.

[33] Butler, N.S., et al., Superior antimalarial immunity after vaccination with late liver stagearresting genetically attenuated parasites. Cell Host Microbe, 2011. 9(6): p. 451-62.

[34] Woodberry, T., et al., Human T cell recognition of the blood stage antigen Plasmodium hypoxanthine guanine xanthine phosphoribosyl transferase (HGXPRT) in acute malaria. Malar J, 2009. 8: p. 122.

[35] Good, M.F., A whole parasite vaccine to control the blood stages of Plasmodium: the case for lateral thinking. Trends Parasitol, 2011. 27(8): p. 335-40.

[36] Richards, J.S. and J.G. Beeson, The future for blood-stage vaccines against malaria. Immunol Cell Biol, 2009. 87(5): p. 377-90.

[37] Moorthy, V.S., M.F. Good, and A.V. Hill, Malaria vaccine developments. Lancet, 2004. 363(9403): p. 150-6.

[38] Arevalo-Herrera, M., C. Chitnis, and S. Herrera, Current status of Plasmodium vivax vaccine. Hum Vaccin, 2010. 6(1): p. 124-32.

[39] Galinski, M.R. and J.W. Barnwell, Plasmodium vivax: who cares? Malar J, 2008. 7 Suppl 1: p. S9.

[40] Crabb, B.S. and A.F. Cowman, Characterization of promoters and stable transfection by homologous and nonhomologous recombination in Plasmodium falciparum. Proc Natl Acad Sci U S A, 1996. 93(14): p. 7289-94.

[41] Wu, Y., L.A. Kirkman, and T.E. Wellems, Transformation of Plasmodium falciparum malaria parasites by homologous integration of plasmids that confer resistance to pyrimethamine. Proc Natl Acad Sci U S A, 1996. 93(3): p. 1130-4.

[42] Wu, Y., et al., Transfection of Plasmodium falciparum within human red blood cells. Proc Natl Acad Sci U S A, 1995. 92(4): p. 973-7.

[43] Alonso, P.L., et al., Duration of protection with RTS,S/AS02A malaria vaccine in prevention of Plasmodium falciparum disease in Mozambican children: single-blind extended follow-up of a randomised controlled trial. Lancet, 2005. 366(9502): p. 2012-8.

[44] Alonso, P.L., et al., Efficacy of the RTS,S/AS02A vaccine against Plasmodium falciparum infection and disease in young African children: randomised controlled trial. Lancet, 2004. 364(9443): p. 1411-20.

[45] Sirima, S.B., S. Cousens, and P. Druilhe, Protection against malaria by MSP3 candidate vaccine. N Engl J Med, 2011. 365(11): p. 1062-4.

[46] Thera, M.A., et al., A field trial to assess a blood-stage malaria vaccine. N Engl J Med, 2011. 365(11): p. 1004-13. 
[47] Volkman, S.K., et al., A genome-wide map of diversity in Plasmodium falciparum. Nat Genet, 2007. 39(1): p. 113-9.

[48] Doolan, D.L., Plasmodium immunomics. Int J Parasitol, 2010.

[49] Sanders, P.R., et al., Distinct protein classes including novel merozoite surface antigens in Raft-like membranes of Plasmodium falciparum. J Biol Chem, 2005. 280(48): p. 40169-76.

[50] Arumugam, T.U., et al., Discovery of GAMA, a Plasmodium falciparum merozoite micronemal protein, as a novel blood-stage vaccine candidate antigen. Infect Immun, 2011. 79(11): p. 4523-32.

[51] Organisation, W.H. Malaria Vaccine Rainbow Tables. . 2010 December 2010; Available from: http://www.who.int/vaccine_research/links/Rainbow/en/index.html.

[52] Snow, R.W., et al., The global distribution of clinical episodes of Plasmodium falciparum malaria. Nature, 2005. 434(7030): p. 214-7.

[53] Enea, V., et al., DNA cloning of Plasmodium falciparum circumsporozoite gene: amino acid sequence of repetitive epitope. Science, 1984. 225(4662): p. 628-30.

[54] Dame, J.B., et al., Structure of the gene encoding the immunodominant surface antigen on the sporozoite of the human malaria parasite Plasmodium falciparum. Science, 1984. 225(4662): p. 593-9.

[55] Good, M.F., et al., Human T-cell recognition of the circumsporozoite protein of Plasmodium falciparum: immunodominant T-cell domains map to the polymorphic regions of the molecule. Proc Natl Acad Sci U S A, 1988. 85(4): p. 1199-203.

[56] First Results of Phase 3 Trial of RTS,S/AS01 Malaria Vaccine in African Children. N Engl J Med, 2011.

[57] Cowan, G., et al., Expression of thrombospondin-related anonymous protein in Plasmodium falciparum sporozoites. Lancet, 1992. 339(8806): p. 1412-3.

[58] Ghosh, A.K., et al., Malaria parasite invasion of the mosquito salivary gland requires interaction between the Plasmodium TRAP and the Anopheles saglin proteins. PLoS Pathog, 2009. 5(1): p. e1000265.

[59] Sultan, A.A., et al., TRAP is necessary for gliding motility and infectivity of plasmodium sporozoites. Cell, 1997. 90(3): p. 511-22.

[60] Kappe, S., et al., Conservation of a gliding motility and cell invasion machinery in Apicomplexan parasites. J Cell Biol, 1999. 147(5): p. 937-44.

[61] Charoenvit, Y., et al., Development of two monoclonal antibodies against Plasmodium falciparum sporozoite surface protein 2 and mapping of B-cell epitopes. Infect Immun, 1997. 65(8): p. 3430-7.

[62] Wizel, B., et al., Irradiated sporozoite vaccine induces HLA-B8-restricted cytotoxic T lymphocyte responses against two overlapping epitopes of the Plasmodium falciparum sporozoite surface protein 2. J Exp Med, 1995. 182(5): p. 1435-45.

[63] Wizel, B., et al., HLA-A2-restricted cytotoxic T lymphocyte responses to multiple Plasmodium falciparum sporozoite surface protein 2 epitopes in sporozoite-immunized volunteers. J Immunol, 1995. 155(2): p. 766-75.

[64] Aidoo, M., et al., Identification of conserved antigenic components for a cytotoxic $T$ lymphocyte-inducing vaccine against malaria. Lancet, 1995. 345(8956): p. 1003-7.

[65] Robson, K.J., et al., Natural polymorphism in the thrombospondin-related adhesive protein of Plasmodium falciparum. Am J Trop Med Hyg, 1998. 58(1): p. 81-9.

[66] Weedall, G.D., et al., Differential evidence of natural selection on two leading sporozoite stage malaria vaccine candidate antigens. Int J Parasitol, 2007. 37(1): p. 77-85. 
[67] Gilbert, S.C., et al., Synergistic DNA-MVA prime-boost vaccination regimes for malaria and tuberculosis. Vaccine, 2006. 24(21): p. 4554-61.

[68] McConkey, S.J., et al., Enhanced T-cell immunogenicity of plasmid DNA vaccines boosted by recombinant modified vaccinia virus Ankara in humans. Nat Med, 2003. 9(6): p. 729-35.

[69] Bejon, P., et al., Calculation of liver-to-blood inocula, parasite growth rates, and preerythrocytic vaccine efficacy, from serial quantitative polymerase chain reaction studies of volunteers challenged with malaria sporozoites. J Infect Dis, 2005. 191(4): p. 619-26.

[70] Bejon, P., et al., A phase $2 b$ randomised trial of the candidate malaria vaccines FP9 ME-TRAP and MVA ME-TRAP among children in Kenya. PLoS Clin Trials, 2006. 1(6): p. e29.

[71] Bejon, P., et al., Extended follow-up following a phase $2 b$ randomized trial of the candidate malaria vaccines FP9 ME-TRAP and MVA ME-TRAP among children in Kenya. PLoS One, 2007. 2(8): p. e707.

[72] Moorthy, V.S., et al., A randomised, double-blind, controlled vaccine efficacy trial of DNA/MVA ME-TRAP against malaria infection in Gambian adults. PLoS Med, 2004. 1(2): p. e33.

[73] Moorthy, V.S., et al., Phase 1 evaluation of 3 highly immunogenic prime-boost regimens, including a 12-month reboosting vaccination, for malaria vaccination in Gambian men. J Infect Dis, 2004. 189(12): p. 2213-9.

[74] Peterson, M.G., et al., Integral membrane protein located in the apical complex of Plasmodium falciparum. Mol Cell Biol, 1989. 9(7): p. 3151-4.

[75] Narum, D.L. and A.W. Thomas, Differential localization of full-length and processed forms of PF83/AMA-1 an apical membrane antigen of Plasmodium falciparum merozoites. Mol Biochem Parasitol, 1994. 67(1): p. 59-68.

[76] Howell, S.A., et al., Proteolytic processing and primary structure of Plasmodium falciparum apical membrane antigen-1. J Biol Chem, 2001. 276(33): p. 31311-20.

[77] Howell, S.A., et al., A single malaria merozoite serine protease mediates shedding of multiple surface proteins by juxtamembrane cleavage. J Biol Chem, 2003. 278(26): p. 23890-8.

[78] Collins, C.R., et al., An inhibitory antibody blocks interactions between components of the malarial invasion machinery. PLoS Pathog, 2009. 5(1): p. e1000273.

[79] Besteiro, S., et al., Export of a Toxoplasma gondii rhoptry neck protein complex at the host cell membrane to form the moving junction during invasion. PLoS Pathog, 2009. 5(2): p. e1000309.

[80] Silvie, O., et al., Malaria sporozoite: migrating for a living. Trends Mol Med, 2004. 10(3): p. 97-100; discussion 100-1.

[81] Johnson, A.H., et al., Human leukocyte antigen class II alleles influence levels of antibodies to the Plasmodium falciparum asexual-stage apical membrane antigen 1 but not to merozoite surface antigen 2 and merozoite surface protein 1. Infect Immun, 2004. 72(5): p. 2762-71.

[82] Thomas, A.W., et al., Aspects of immunity for the AMA-1 family of molecules in humans and non-human primates malarias. Mem Inst Oswaldo Cruz, 1994. 89 Suppl 2: p. 67-70.

[83] Cortes, A., et al., Allele specificity of naturally acquired antibody responses against Plasmodium falciparum apical membrane antigen 1. Infect Immun, 2005. 73(1): p. 42230.

[84] Hodder, A.N., P.E. Crewther, and R.F. Anders, Specificity of the protective antibody response to apical membrane antigen 1. Infect Immun, 2001. 69(5): p. 3286-94. 
[85] Kennedy, M.C., et al., In vitro studies with recombinant Plasmodium falciparum apical membrane antigen 1 (AMA1): production and activity of an AMA1 vaccine and generation of a multiallelic response. Infect Immun, 2002. 70(12): p. 6948-60.

[86] Kocken, C.H., et al., High-level expression of the malaria blood-stage vaccine candidate Plasmodium falciparum apical membrane antigen 1 and induction of antibodies that inhibit erythrocyte invasion. Infect Immun, 2002. 70(8): p. 4471-6.

[87] Yoshida, S., et al., Plasmodium berghei circumvents immune responses induced by merozoite surface protein 1- and apical membrane antigen 1-based vaccines. PLoS One, 2010. 5(10): p. e13727.

[88] Miura, K., et al., In immunization with Plasmodium falciparum apical membrane antigen 1, the specificity of antibodies depends on the species immunized. Infect Immun, 2007. 75(12): p. 5827-36.

[89] Narum, D.L., et al., Passive immunization with a multicomponent vaccine against conserved domains of apical membrane antigen 1 and 235-kilodalton rhoptry proteins protects mice against Plasmodium yoelii blood-stage challenge infection. Infect Immun, 2006. 74(10): p. 5529-36.

[90] Barclay, V.C., et al., Mixed allele malaria vaccines: host protection and within-host selection. Vaccine, 2008. 26(48): p. 6099-107.

[91] Duan, J., et al., Population structure of the genes encoding the polymorphic Plasmodium falciparum apical membrane antigen 1: implications for vaccine design. Proc Natl Acad Sci U S A, 2008. 105(22): p. 7857-62.

[92] Takala, S.L., et al., Extreme polymorphism in a vaccine antigen and risk of clinical malaria: implications for vaccine development. Sci Transl Med, 2009. 1(2): p. 2 ra5.

[93] Cortes, A., et al., Geographical structure of diversity and differences between symptomatic and asymptomatic infections for Plasmodium falciparum vaccine candidate AMA1. Infect Immun, 2003. 71(3): p. 1416-26.

[94] Kusi, K.A., et al., Generation of humoral immune responses to multi-allele PfAMA1 vaccines; effect of adjuvant and number of component alleles on the breadth of response. PLoS One, 2010. 5(11): p. e15391.

[95] Kusi, K.A., et al., Humoral immune response to mixed PfAMA1 alleles; multivalent PfAMA1 vaccines induce broad specificity. PLoS One, 2009. 4(12): p. e8110.

[96] Dutta, S., et al., Alanine mutagenesis of the primary antigenic escape residue cluster, c1, of apical membrane antigen 1. Infect Immun, 2010. 78(2): p. 661-71.

[97] Dutta, S., et al., Structural basis of antigenic escape of a malaria vaccine candidate. Proc Natl Acad Sci U S A, 2007. 104(30): p. 12488-93.

[98] Ouattara, A., et al., Lack of allele-specific efficacy of a bivalent AMA1 malaria vaccine. Malar J, 2010. 9: p. 175.

[99] Remarque, E.J., et al., A diversity-covering approach to immunization with Plasmodium falciparum apical membrane antigen 1 induces broader allelic recognition and growth inhibition responses in rabbits. Infect Immun, 2008. 76(6): p. 2660-70.

[100] McBride, J.S. and H.G. Heidrich, Fragments of the polymorphic Mr 185,000 glycoprotein from the surface of isolated Plasmodium falciparum merozoites form an antigenic complex. Mol Biochem Parasitol, 1987. 23(1): p. 71-84.

[101] Gerold, P., et al., Structural analysis of the glycosyl-phosphatidylinositol membrane anchor of the merozoite surface proteins-1 and -2 of Plasmodium falciparum. Mol Biochem Parasitol, 1996. 75(2): p. 131-43. 
[102] Barale, J.C., et al., Plasmodium falciparum subtilisin-like protease 2, a merozoite candidate for the merozoite surface protein 1-42 maturase. Proc Natl Acad Sci U S A, 1999. 96(11): p. 6445-50.

[103] Hackett, F., et al., PfSUB-2: a second subtilisin-like protein in Plasmodium falciparum merozoites. Mol Biochem Parasitol, 1999. 103(2): p. 183-95.

[104] Harris, P.K., et al., Molecular identification of a malaria merozoite surface sheddase. PLoS Pathog, 2005. 1(3): p. 241-51.

[105] Blackman, M.J., et al., Antibodies inhibit the protease-mediated processing of a malaria merozoite surface protein. J Exp Med, 1994. 180(1): p. 389-93.

[106] Blackman, M.J., et al., A single fragment of a malaria merozoite surface protein remains on the parasite during red cell invasion and is the target of invasion-inhibiting antibodies. J Exp Med, 1990. 172(1): p. 379-82.

[107] Tanabe, K., et al., Allelic dimorphism in a surface antigen gene of the malaria parasite Plasmodium falciparum. J Mol Biol, 1987. 195(2): p. 273-87.

[108] Miller, L.H., et al., Analysis of sequence diversity in the Plasmodium falciparum merozoite surface protein-1 (MSP-1). Mol Biochem Parasitol, 1993. 59(1): p. 1-14.

[109] Da Silveira, L.A., et al., Sequence diversity and linkage disequilibrium within the merozoite surface protein-1 (Msp-1) locus of Plasmodium falciparum: a longitudinal study in Brazil. J Eukaryot Microbiol, 2001. 48(4): p. 433-9.

[110] Ferreira, M.U., et al., Sequence diversity and evolution of the malaria vaccine candidate merozoite surface protein-1 (MSP-1) of Plasmodium falciparum. Gene, 2003. 304: p. 6575.

[111] Qari, S.H., et al., Predicted and observed alleles of Plasmodium falciparum merozoite surface protein-1 (MSP-1), a potential malaria vaccine antigen. Mol Biochem Parasitol, 1998. 92(2): p. 241-52.

[112] Sakihama, N., et al., Allelic recombination and linkage disequilibrium within Msp-1 of Plasmodium falciparum, the malignant human malaria parasite. Gene, 1999. 230(1): p. 4754.

[113] Takala, S.L., et al., Dynamics of polymorphism in a malaria vaccine antigen at a vaccinetesting site in Mali. PLoS Med, 2007. 4(3): p. e93.

[114] Thera, M.A., et al., Safety and allele-specific immunogenicity of a malaria vaccine in Malian adults: results of a phase I randomized trial. PLoS Clin Trials, 2006. 1(7): p. e34.

[115] Ogutu, B.R., et al., Blood stage malaria vaccine eliciting high antigen-specific antibody concentrations confers no protection to young children in Western Kenya. PLoS One, 2009. 4(3): p. e4708.

[116] Goodman, A.L., et al., New candidate vaccines against blood-stage Plasmodium falciparum malaria: prime-boost immunization regimens incorporating human and simian adenoviral vectors and poxviral vectors expressing an optimized antigen based on merozoite surface protein 1. Infect Immun, 2010. 78(11): p. 4601-12.

[117] Fenton, B., et al., Structural and antigenic polymorphism of the 35- to 48-kilodalton merozoite surface antigen (MSA-2) of the malaria parasite Plasmodium falciparum. Mol Cell Biol, 1991. 11(2): p. 963-71.

[118] Smythe, J.A., et al., Structural diversity in the Plasmodium falciparum merozoite surface antigen 2. Proc Natl Acad Sci U S A, 1991. 88(5): p. 1751-5.

[119] Smythe, J.A., et al., Structural diversity in the 45-kilodalton merozoite surface antigen of Plasmodium falciparum. Mol Biochem Parasitol, 1990. 39(2): p. 227-34. 
[120] Snewin, V.A., et al., Polymorphism of the alleles of the merozoite surface antigens MSA1 and MSA2 in Plasmodium falciparum wild isolates from Colombia. Mol Biochem Parasitol, 1991. 49(2): p. 265-75.

[121] Falk, N., et al., Comparison of PCR-RFLP and Genescan-based genotyping for analyzing infection dynamics of Plasmodium falciparum. Am J Trop Med Hyg, 2006. 74(6): p. 94450.

[122] Schoepflin, S., et al., Comparison of Plasmodium falciparum allelic frequency distribution in different endemic settings by high-resolution genotyping. Malar J, 2009. 8: p. 250.

[123] Ranford-Cartwright, L.C., et al., Differential antibody recognition of FC27-like Plasmodium falciparum merozoite surface protein MSP2 antigens which lack 12 amino acid repeats. Parasite Immunol, 1996. 18(8): p. 411-20.

[124] Taylor, R.R., et al., Human antibody response to Plasmodium falciparum merozoite surface protein 2 is serogroup specific and predominantly of the immunoglobulin G3 subclass. Infect Immun, 1995. 63(11): p. 4382-8.

[125] Stanisic, D.I., et al., Immunoglobulin G subclass-specific responses against Plasmodium falciparum merozoite antigens are associated with control of parasitemia and protection from symptomatic illness. Infect Immun, 2009. 77(3): p. 1165-74.

[126] Taylor, R.R., et al., IgG3 antibodies to Plasmodium falciparum merozoite surface protein 2 (MSP2): increasing prevalence with age and association with clinical immunity to malaria. Am J Trop Med Hyg, 1998. 58(4): p. 406-13.

[127] Metzger, W.G., et al., Serum IgG3 to the Plasmodium falciparum merozoite surface protein 2 is strongly associated with a reduced prospective risk of malaria. Parasite Immunol, 2003. 25(6): p. 307-12.

[128] al-Yaman, F., et al., Assessment of the role of the humoral response to Plasmodium falciparum MSP2 compared to RESA and SPf66 in protecting Papua New Guinean children from clinical malaria. Parasite Immunol, 1995. 17(9): p. 493-501.

[129] Bruce, M.C., et al., Genetic diversity and dynamics of plasmodium falciparum and P. vivax populations in multiply infected children with asymptomatic malaria infections in Papua New Guinea. Parasitology, 2000. 121 ( Pt 3): p. 257-72.

[130] Franks, S., et al., Frequent and persistent, asymptomatic Plasmodium falciparum infections in African infants, characterized by multilocus genotyping. J Infect Dis, 2001. 183(5): p. 796804.

[131] Weisman, S., et al., Antibody responses to infections with strains of Plasmodium falciparum expressing diverse forms of merozoite surface protein 2. Infect Immun, 2001. 69(2): p. 959-67.

[132] McCarthy, J.S., et al., A pilot randomised trial of induced blood-stage Plasmodium falciparum infections in healthy volunteers for testing efficacy of new antimalarial drugs. PLoS One, 2011. 6(8): p. e21914.

[133] McColl, D.J., et al., Molecular variation in a novel polymorphic antigen associated with Plasmodium falciparum merozoites. Mol Biochem Parasitol, 1994. 68(1): p. 53-67.

[134] McColl, D.J. and R.F. Anders, Conservation of structural motifs and antigenic diversity in the Plasmodium falciparum merozoite surface protein-3 (MSP-3). Mol Biochem Parasitol, 1997. 90(1): p. 21-31.

[135] Oeuvray, C., et al., Merozoite surface protein-3: a malaria protein inducing antibodies that promote Plasmodium falciparum killing by cooperation with blood monocytes. Blood, 1994. 84(5): p. 1594-602. 
[136] Huber, W., et al., Limited sequence polymorphism in the Plasmodium falciparum merozoite surface protein 3. Mol Biochem Parasitol, 1997. 87(2): p. 231-4.

[137] Okenu, D.M., A.W. Thomas, and D.J. Conway, Allelic lineages of the merozoite surface protein 3 gene in Plasmodium reichenowi and Plasmodium falciparum. Mol Biochem Parasitol, 2000. 109(2): p. 185-8.

[138] Polley, S.D., et al., Plasmodium falciparum merozoite surface protein 3 is a target of allelespecific immunity and alleles are maintained by natural selection. J Infect Dis, 2007. 195(2): p. 279-87.

[139] Audran, R., et al., Phase I malaria vaccine trial with a long synthetic peptide derived from the merozoite surface protein 3 antigen. Infect Immun, 2005. 73(12): p. 8017-26.

[140] Mordmuller, B., et al., Safety and immunogenicity of the malaria vaccine candidate GMZ2 in malaria-exposed, adult individuals from Lambarene, Gabon. Vaccine, 2010. 28(41): p. 6698-703.

[141] Camus, D. and T.J. Hadley, A Plasmodium falciparum antigen that binds to host erythrocytes and merozoites. Science, 1985. 230(4725): p. 553-6.

[142] Sim, B.K., et al., Primary structure of the 175K Plasmodium falciparum erythrocyte binding antigen and identification of a peptide which elicits antibodies that inhibit malaria merozoite invasion. J Cell Biol, 1990. 111(5 Pt 1): p. 1877-84.

[143] Sim, B.K., et al., Receptor and ligand domains for invasion of erythrocytes by Plasmodium falciparum. Science, 1994. 264(5167): p. 1941-4.

[144] Tolia, N.H., et al., Structural basis for the EBA-175 erythrocyte invasion pathway of the malaria parasite Plasmodium falciparum. Cell, 2005. 122(2): p. 183-93.

[145] Okenu, D.M., et al., Analysis of human antibodies to erythrocyte binding antigen 175 of Plasmodium falciparum. Infect Immun, 2000. 68(10): p. 5559-66.

[146] Okoyeh, J.N., C.R. Pillai, and C.E. Chitnis, Plasmodium falciparum field isolates commonly use erythrocyte invasion pathways that are independent of sialic acid residues of glycophorin A. Infect Immun, 1999. 67(11): p. 5784-91.

[147] Richards, J.S., et al., Association between naturally acquired antibodies to erythrocyte-binding antigens of Plasmodium falciparum and protection from malaria and high-density parasitemia. Clin Infect Dis, 2010. 51(8): p. e50-60.

[148] Narum, D.L., et al., Antibodies against the Plasmodium falciparum receptor binding domain of EBA-175 block invasion pathways that do not involve sialic acids. Infect Immun, 2000. 68(4): p. 1964-6.

[149] Baum, J., A.W. Thomas, and D.J. Conway, Evidence for diversifying selection on erythrocyte-binding antigens of Plasmodium falciparum and P. vivax. Genetics, 2003. 163(4): p. 1327-36.

[150] Persson, K.E., et al., Variation in use of erythrocyte invasion pathways by Plasmodium falciparum mediates evasion of human inhibitory antibodies. J Clin Invest, 2008. 118(1): p. 342-51.

[151] Jones, T.R., et al., Protection of Aotus monkeys by Plasmodium falciparum EBA-175 region II DNA prime-protein boost immunization regimen. J Infect Dis, 2001. 183(2): p. 303-312.

[152] El Sahly, H.M., et al., Safety and immunogenicity of a recombinant nonglycosylated erythrocyte binding antigen 175 Region II malaria vaccine in healthy adults living in an area where malaria is not endemic. Clin Vaccine Immunol, 2010. 17(10): p. 1552-9.

[153] Rener, J., et al., Target antigens of transmission-blocking immunity on gametes of plasmodium falciparum. J Exp Med, 1983. 158(3): p. 976-81. 
[154] Vermeulen, A.N., et al., Sequential expression of antigens on sexual stages of Plasmodium falciparum accessible to transmission-blocking antibodies in the mosquito. J Exp Med, 1985. 162(5): p. 1460-76.

[155] Quakyi, I.A., et al., The 230-kDa gamete surface protein of Plasmodium falciparum is also a target for transmission-blocking antibodies. J Immunol, 1987. 139(12): p. 4213-7.

[156] Barr, P.J., et al., Recombinant Pfs25 protein of Plasmodium falciparum elicits malaria transmission-blocking immunity in experimental animals. J Exp Med, 1991. 174(5): p. 1203-8.

[157] Kocken, C.H., et al., Cloning and expression of the gene coding for the transmission blocking target antigen Pfs48/45 of Plasmodium falciparum. Mol Biochem Parasitol, 1993. 61(1): p. 59-68.

[158] Kaslow, D.C., et al., Comparison of the primary structure of the $25 \mathrm{kDa}$ ookinete surface antigens of Plasmodium falciparum and Plasmodium gallinaceum reveal six conserved regions. Mol Biochem Parasitol, 1989. 33(3): p. 283-7.

[159] Shi, Y.P., et al., Single amino acid variation in the ookinete vaccine antigen from field isolates of Plasmodium falciparum. Mol Biochem Parasitol, 1992. 50(1): p. 179-80.

[160] Kaslow, D.C., et al., A vaccine candidate from the sexual stage of human malaria that contains EGF-like domains. Nature, 1988. 333(6168): p. 74-6.

[161] van Dijk, M.R., et al., A central role for P48/45 in malaria parasite male gamete fertility. Cell, 2001. 104(1): p. 153-64.

[162] Roeffen, W., et al., Association between anti-Pfs48/45 reactivity and $P$. falciparum transmission-blocking activity in sera from Cameroon. Parasite Immunol, 1996. 18(2): p. 103-9.

[163] Duffy, P.E. and D.C. Kaslow, A novel malaria protein, Pfs28, and Pfs25 are genetically linked and synergistic as falciparum malaria transmission-blocking vaccines. Infect Immun, 1997. 65(3): p. 1109-13.

[164] Anthony, T.G., et al., Evidence of non-neutral polymorphism in Plasmodium falciparum gamete surface protein genes $P f_{s} 47$ and Pfs48/45. Mol Biochem Parasitol, 2007. 156(2): p. 117-23.

[165] Conway, D.J., et al., Extreme geographical fixation of variation in the Plasmodium falciparum gamete surface protein gene Pfs $48 / 45$ compared with microsatellite loci. Mol Biochem Parasitol, 2001. 115(2): p. 145-56.

[166] Wu, Y., et al., Phase 1 trial of malaria transmission blocking vaccine candidates Pfs 25 and Pvs25 formulated with montanide ISA 51. PLoS One, 2008. 3(7): p. e2636.

[167] Kubler-Kielb, J., et al., Long-lasting and transmission-blocking activity of antibodies to Plasmodium falciparum elicited in mice by protein conjugates of Pfs25. Proc Natl Acad Sci U S A, 2007. 104(1): p. 293-8.

[168] Reyes-Sandoval, A., et al., Prime-boost immunization with adenoviral and modified vaccinia virus Ankara vectors enhances the durability and polyfunctionality of protective malaria CD8+ T-cell responses. Infect Immun, 2010. 78(1): p. 145-53.

[169] Hillier, C.J., et al., Process development and analysis of liver-stage antigen 1, a preerythrocytestage protein-based vaccine for Plasmodium falciparum. Infect Immun, 2005. 73(4): p. 2109-15.

[170] Goschnick, M.W., et al., Merozoite surface protein 4/5 provides protection against lethal challenge with a heterologous malaria parasite strain. Infect Immun, 2004. 72(10): p. 5840-9. 
[171] Reiling, L., et al., Evidence that the erythrocyte invasion ligand PfRh2 is a target of protective immunity against Plasmodium falciparum malaria. J Immunol, 2010. 185(10): p. 615767.

[172] Lopaticki, S., et al., Reticulocyte and erythrocyte binding-like proteins function cooperatively in invasion of human erythrocytes by malaria parasites. Infect Immun, 2011. 79(3): p. 1107-17.

[173] Chen, L., et al., An EGF-like protein forms a complex with PfRh5 and is required for invasion of human erythrocytes by Plasmodium falciparum. PLoS Pathog, 2011. 7(9): p. e1002199.

[174] Palacpac, N.M., et al., Plasmodium falciparum serine repeat antigen 5 (SE36) as a malaria vaccine candidate. Vaccine, 2011. 29(35): p. 5837-45.

[175] Demanga, C.G., et al., Toward the rational design of a malaria vaccine construct using the MSP3 family as an example: contribution of antigenicity studies in humans. Infect Immun, 2010. 78(1): p. 486-94.

[176] Pachebat, J.A., et al., Extensive proteolytic processing of the malaria parasite merozoite surface protein 7 during biosynthesis and parasite release from erythrocytes. Mol Biochem Parasitol, 2007. 151(1): p. 59-69.

[177] Barfod, L., et al., Baculovirus-expressed constructs induce immunoglobulin $G$ that recognizes VAR2CSA on Plasmodium falciparum-infected erythrocytes. Infect Immun, 2006. 74(7): p. $4357-60$.

[178] Salanti, A., et al., Several domains from VAR2CSA can induce Plasmodium falciparum adhesion-blocking antibodies. Malar J, 2010. 9: p. 11.

[179] Srivastava, A., et al., Full-length extracellular region of the var2CSA variant of PfEMP1 is required for specific, high-affinity binding to CSA. Proc Natl Acad Sci U S A, 2010. 107(11): p. 4884-9.

[180] Snounou, G. and L. Renia, The vaccine is dead--long live the vaccine. Trends Parasitol, 2007. 23(4): p. 129-32.

[181] Tham, W.H., Healer, J.,Cowman, A.F., Erythrocyte and reticulocyte binding-like proteins of Plasmodium falciparum. Trends in Parasitology, 2011. In press.

[182] Mueller, I., et al., Key gaps in the knowledge of Plasmodium vivax, a neglected human malaria parasite. Lancet Infect Dis, 2009. 9(9): p. 555-66.

[183] Chitnis, C.E. and A. Sharma, Targeting the Plasmodium vivax Duffy-binding protein. Trends Parasitol, 2008. 24(1): p. 29-34.

[184] Miller, L.H., et al., The resistance factor to Plasmodium vivax in blacks. The Duffy-bloodgroup genotype, FyFy. N Engl J Med, 1976. 295(6): p. 302-4.

[185] Gentil, F., et al., A recombinant vaccine based on domain II of Plasmodium vivax Apical Membrane Antigen 1 induces high antibody titres in mice. Vaccine, 2010. 28(38): p. 618390.

[186] Castellanos, A., et al., Plasmodium vivax thrombospondin related adhesion protein: immunogenicity and protective efficacy in rodents and Aotus monkeys. Mem Inst Oswaldo Cruz, 2007. 102(3): p. 411-6.

[187] Putaporntip, C., et al., Mosaic organization and heterogeneity in frequency of allelic recombination of the Plasmodium vivax merozoite surface protein-1 locus. Proc Natl Acad Sci U S A, 2002. 99(25): p. 16348-53.

[188] Cheng, Q. and A. Saul, Sequence analysis of the apical membrane antigen I (AMA-1) of Plasmodium vivax. Mol Biochem Parasitol, 1994. 65(1): p. 183-7. 
[189] Lima-Junior, J.C., et al., Promiscuous T-cell epitopes of Plasmodium merozoite surface protein 9 (PvMSP9) induces IFN-gamma and IL-4 responses in individuals naturally exposed to malaria in the Brazilian Amazon. Vaccine, 2010. 28(18): p. 3185-91.

[190] Marsh, K. and S. Kinyanjui, Immune effector mechanisms in malaria. Parasite Immunol, 2006. 28(1-2): p. 51-60.

[191] Gupta, S., et al., Immunity to non-cerebral severe malaria is acquired after one or two infections. Nat Med, 1999. 5(3): p. 340-3.

[192] Fowkes, F.J., et al., The relationship between anti-merozoite antibodies and incidence of Plasmodium falciparum malaria: A systematic review and meta-analysis. PLoS Med, 2010. 7(1): p. e1000218.

[193] Scopel, K.K., et al., Variant-specific antibodies to merozoite surface protein 2 and clinical expression of Plasmodium falciparum malaria in rural Amazonians. Am J Trop Med Hyg, 2007. 76(6): p. 1084-91.

[194] Osier, F.H., et al., Allele-specific antibodies to Plasmodium falciparum merozoite surface protein-2 and protection against clinical malaria. Parasite Immunol, 2010. 32(3): p. 193201.

[195] Kusi, K.A., et al., Safety and immunogenicity of multi-antigen AMA1-based vaccines formulated with CoVaccine HT and Montanide ISA 51 in rhesus macaques. Malar J, 2011. 10: p. 182.

[196] Druilhe, P., et al., A malaria vaccine that elicits in humans antibodies able to kill Plasmodium falciparum. PLoS Med, 2005. 2(11): p. e344.

[197] Waitumbi, J.N., et al., Impact of RTS,S/AS02(A) and RTS,S/AS01(B) on genotypes of P. falciparum in adults participating in a malaria vaccine clinical trial. PLoS One, 2009. 4(11): p. e7849.

[198] Enosse, S., et al., RTS,S/AS02A malaria vaccine does not induce parasite CSP T cell epitope selection and reduces multiplicity of infection. PLoS Clin Trials, 2006. 1(1): p. e5.

[199] Beeson, J.G. and B.S. Crabb, Towards a vaccine against Plasmodium vivax malaria. PLoS Med, 2007. 4(12): p. e350.

[200] VanBuskirk, K.M., et al., Antigenic drift in the ligand domain of Plasmodium vivax duffy binding protein confers resistance to inhibitory antibodies. J Infect Dis, 2004. 190(9): p. 1556-62.

[201] Cole-Tobian, J.L., et al., Strain-specific duffy binding protein antibodies correlate with protection against infection with homologous compared to heterologous plasmodium vivax strains in Papua New Guinean children. Infect Immun, 2009. 77(9): p. 4009-17.

[202] Verra, F., et al., Contrasting signatures of selection on the Plasmodium falciparum erythrocyte binding antigen gene family. Mol Biochem Parasitol, 2006. 149(2): p. 182-90.

[203] Mamillapalli, A., et al., Polymorphism and epitope sharing between the alleles of merozoite surface protein-1 of Plasmodium falciparum among Indian isolates. Malar J, 2007. 6: p. 95.

[204] Jiang, L., et al., Evidence for erythrocyte-binding antigen 175 as a component of a ligandblocking blood-stage malaria vaccine. Proc Natl Acad Sci U S A, 2011. 108(18): p. 75538.

[205] Barry, A.E., et al., Population genomics of the immune evasion (var) genes of Plasmodium falciparum. PLoS Pathog, 2007. 3(3): p. e34.

[206] Chen, D.S., et al., A molecular epidemiological study of var gene diversity to characterize the reservoir of Plasmodium falciparum in humans in Africa. PLoS One, 2011. 6(2): p. e16629. 
[207] Smith, J.D., et al., Switches in expression of Plasmodium falciparum var genes correlate with changes in antigenic and cytoadherent phenotypes of infected erythrocytes. Cell, 1995. 82(1): p. 101-10.

[208] Baruch, D.I., et al., Immunization of Aotus monkeys with a functional domain of the Plasmodium falciparum variant antigen induces protection against a lethal parasite line. Proc Natl Acad Sci U S A, 2002. 99(6): p. 3860-5.

[209] Salanti, A., et al., Evidence for the involvement of VAR2CSA in pregnancy-associated malaria. J Exp Med, 2004. 200(9): p. 1197-203.

[210] Trimnell, A.R., et al., Global genetic diversity and evolution of var genes associated with placental and severe childhood malaria. Mol Biochem Parasitol, 2006.

[211] Hommel, M., et al., Evaluation of the antigenic diversity of placenta-binding Plasmodium falciparum variants and the antibody repertoire among pregnant women. Infect Immun, 2010. 78(5): p. 1963-78.

[212] Bockhorst, J., et al., Structural polymorphism and diversifying selection on the pregnancy malaria vaccine candidate VAR2CSA. Mol Biochem Parasitol, 2007. 155(2): p. 103-12.

[213] Avril, M., et al., Antibodies to a full-length VAR2CSA immunogen are broadly straintranscendent but do not cross-inhibit different placental-type parasite isolates. PLoS One, 2011. 6(2): p. e16622.

[214] Bigey, P., et al., The NTS-DBL2X region of VAR2CSA induces cross-reactive antibodies that inhibit adhesion of several plasmodium falciparum isolates to chondroitin sulfate A. J Infect Dis, 2011. 204(7): p. 1125-33.

[215] Beeson, J.G., et al., Antigenic differences and conservation among placental Plasmodium falciparum-infected erythrocytes and acquisition of variant-specific and cross-reactive antibodies. J Infect Dis, 2006. 193(5): p. 721-30.

[216] Hartl, D.L., Clark, A.G. , Principles of Population Genetics. 3 ed. 1997, Sunderland, Maryland: Sinauer Associates.

[217] Conway, D.J., Natural selection on polymorphic malaria antigens and the search for a vaccine. Parasitol Today, 1997. 13(1): p. 26-9.

[218] Tetteh, K.K., et al., Prospective identification of malaria parasite genes under balancing selection. PLoS One, 2009. 4(5): p. e5568.

[219] Ochola, L.I., et al., Allele frequency-based and polymorphism-versus-divergence indices of balancing selection in a new filtered set of polymorphic genes in Plasmodium falciparum. Mol Biol Evol, 2010. 27(10): p. 2344-51.

[220] Guerra, C.A., et al., The international limits and population at risk of Plasmodium vivax transmission in 2009. PLoS Negl Trop Dis, 2010. 4(8): p. e774.

[221] Zakeri, S., et al., Circumsporozoite protein gene diversity among temperate and tropical Plasmodium vivax isolates from Iran. Trop Med Int Health, 2006. 11(5): p. 729-37.

[222] Putaporntip, C., et al., Diversity in the thrombospondin-related adhesive protein gene (TRAP) of Plasmodium vivax. Gene, 2001. 268(1-2): p. 97-104.

[223] Ord, R.L., A. Tami, and C.J. Sutherland, ama1 genes of sympatric Plasmodium vivax and P. falciparum from Venezuela differ significantly in genetic diversity and recombination frequency. PLoS One, 2008. 3(10): p. e3366.

[224] Gunasekera, A.M., et al., Genetic diversity and selection at the Plasmodium vivax apical membrane antigen-1 (PvAMA-1) locus in a Sri Lankan population. Mol Biol Evol, 2007. 24(4): p. 939-47.

[225] Moon, S.U., et al., High frequency of genetic diversity of Plasmodium vivax field isolates in Myanmar. Acta Trop, 2009. 109(1): p. 30-6. 
[226] Nobrega de Sousa, T., L.H. Carvalho, and C.F. Alves de Brito, Worldwide genetic variability of the Duffy binding protein: insights into Plasmodium vivax vaccine development. PLoS One, 2011. 6(8): p. e22944.

[227] Zakeri, S., S. Razavi, and N.D. Djadid, Genetic diversity of transmission blocking vaccine candidate (Pvs25 and Pvs28) antigen in Plasmodium vivax clinical isolates from Iran. Acta Trop, 2009. 109(3): p. 176-80.

[228] Hurlbert, S.H., The non-concept of species diversity: a critique and alternative parameters. . Ecology, 1971. 52(577-586).

[229] Excoffier, L. and G. Heckel, Computer programs for population genetics data analysis: a survival guide. Nat Rev Genet, 2006. 7(10): p. 745-58.

[230] Jalloh, A., et al., Sequence variation in the T-cell epitopes of the Plasmodium falciparum circumsporozoite protein among field isolates is temporally stable: a 5-year longitudinal study in southern Vietnam. J Clin Microbiol, 2006. 44(4): p. 1229-35.

[231] Tanabe, K., N. Sakihama, and A. Kaneko, Stable SNPs in malaria antigen genes in isolated populations. Science, 2004. 303(5657): p. 493.

[232] Mu, J., et al., Recombination Hotspots and Population Structure in Plasmodium falciparum. PLoS Biol, 2005. 3(10): p. e335.

[233] Pritchard, J.K., M. Stephens, and P. Donnelly, Inference of population structure using multilocus genotype data. 2000. 155(2): p. 945.

[234] Friedman, S.R. and S. Aral, Social networks, risk-potential networks, health, and disease. J Urban Health, 2001. 78(3): p. 411-8.

[235] Bull, P.C., et al., Plasmodium falciparum antigenic variation. Mapping mosaic var gene sequences onto a network of shared, highly polymorphic sequence blocks. Mol Microbiol, 2008. 68(6): p. 1519-34.

[236] Conway, D.J., et al., A principal target of human immunity to malaria identified by molecular population genetic and immunological analyses. Nat Med, 2000. 6(6): p. 689-92.

[237] Weedall, G.D. and D.J. Conway, Detecting signatures of balancing selection to identify targets of anti-parasite immunity. Trends Parasitol, 2010. 26(7): p. 363-9.

[238] Cui, L., et al., The genetic diversity of Plasmodium vivax populations. Trends Parasitol, 2003. 19(5): p. 220-6.

[239] Innan, H., Modified Hudson-Kreitman-Aguade test and two-dimensional evaluation of neutrality tests. Genetics, 2006. 173(3): p. 1725-33.

[240] Prugnolle, F., et al., African great apes are natural hosts of multiple related malaria species, including Plasmodium falciparum. Proc Natl Acad Sci U S A, 2010. 107(4): p. 1458-63.

[241] Tajima, F., Statistical method for testing the neutral mutation hypothesis by DNA polymorphism. Genetics, 1989. 123(3): p. 585-95.

[242] Polley, S.D., W. Chokejindachai, and D.J. Conway, Allele frequency-based analyses robustly map sequence sites under balancing selection in a malaria vaccine candidate antigen. Genetics, 2003. 165(2): p. 555-61. 


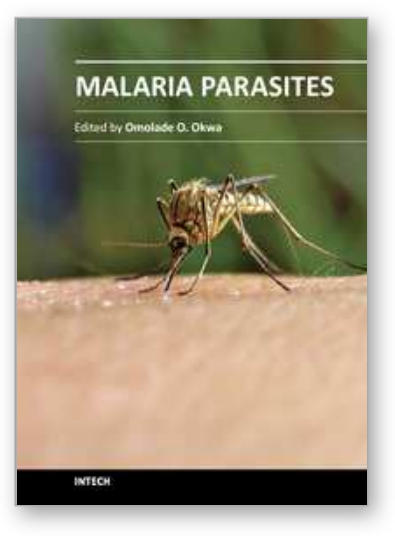

\author{
Malaria Parasites \\ Edited by Dr. Omolade Okwa
}

ISBN 978-953-51-0326-4

Hard cover, 350 pages

Publisher InTech

Published online 30, March, 2012

Published in print edition March, 2012

Malaria is a global disease in the world today but most common in the poorest countries of the world, with $90 \%$ of deaths occurring in sub-Saharan Africa. This book provides information on global efforts made by scientist which cuts across the continents of the world. Concerted efforts such as symbiont based malaria control; new applications in avian malaria studies; development of humanized mice to study P.falciparium (the most virulent species of malaria parasite); and current issues in laboratory diagnosis will support the prompt treatment of malaria. Research is ultimately gaining more grounds in the quest to provide vaccine for the prevention of malaria. The book features research aimed to bring a lasting solution to the malaria problem and what we should be doing now to face malaria, which is definitely useful for health policies in the twenty first century.

\title{
How to reference
}

In order to correctly reference this scholarly work, feel free to copy and paste the following:

Alyssa E. Barry, James Beeson, John C. Reeder, Freya J.I. Fowkes and Alicia Arnott (2012). Using Population Genetics to Guide Malaria Vaccine Design, Malaria Parasites, Dr. Omolade Okwa (Ed.), ISBN: 978-953-510326-4, InTech, Available from: http://www.intechopen.com/books/malaria-parasites/using-populationgenetics-to-guide-malaria-vaccine-design

\section{INTECH}

open science | open minds

\section{InTech Europe}

University Campus STeP Ri

Slavka Krautzeka 83/A

51000 Rijeka, Croatia

Phone: +385 (51) 770447

Fax: +385 (51) 686166

www.intechopen.com

\section{InTech China}

Unit 405, Office Block, Hotel Equatorial Shanghai

No.65, Yan An Road (West), Shanghai, 200040, China

中国上海市延安西路65号上海国际贵都大饭店办公楼405单元

Phone: $+86-21-62489820$

Fax: +86-21-62489821 
(C) 2012 The Author(s). Licensee IntechOpen. This is an open access article distributed under the terms of the Creative Commons Attribution 3.0 License, which permits unrestricted use, distribution, and reproduction in any medium, provided the original work is properly cited. 